\title{
Analysis of a Fractal Boundary: The Graph of the Knopp Function
}

\author{
Mourad Ben Slimane ${ }^{1}$ and Clothilde Mélot $^{2}$ \\ ${ }^{1}$ Department of Mathematics, College of Science, King Saud University, P.O. Box 2455, Riyadh 11451, Saudi Arabia \\ ${ }^{2}$ CNRS, LATP, UMR 6632, Aix-Marseille University, 13453 Marseille, France
}

Correspondence should be addressed to Mourad Ben Slimane; mbenslimane@ksu.edu.sa

Received 26 July 2014; Accepted 16 October 2014

Academic Editor: Weinian Zhang

Copyright (C) 2015 M. Ben Slimane and C. Mélot. This is an open access article distributed under the Creative Commons Attribution License, which permits unrestricted use, distribution, and reproduction in any medium, provided the original work is properly cited.

\begin{abstract}
A usual classification tool to study a fractal interface is the computation of its fractal dimension. But a recent method developed by Y. Heurteaux and S. Jaffard proposes to compute either weak and strong accessibility exponents or local $L^{p}$ regularity exponents (the so-called $p$-exponent). These exponents describe locally the behavior of the interface. We apply this method to the graph of the Knopp function which is defined for $x \in[0,1]$ as $F(x)=\sum_{j=0}^{\infty} 2^{-\alpha j} \phi\left(2^{j} x\right)$, where $0<\alpha<1$ and $\phi(x)=\operatorname{dist}(x, \mathbb{Z})$. The Knopp function itself has everywhere the same $p$-exponent $\alpha$. Nevertheless, using the characterization of the maxima and minima done by B. Dubuc and S. Dubuc, we will compute the $p$-exponent of the characteristic function of the domain under the graph of $F$ at each point $(x, F(x))$ and show that $p$-exponents, weak and strong accessibility exponents, change from point to point. Furthermore we will derive a characterization of the local extrema of the function according to the values of these exponents.
\end{abstract}

\section{Introduction}

At the beginning of the century several examples of nondifferentiable functions were studied, such as the Weiertrass function or the example we will focus on in the following, that is, the Takagi-Knopp or so called Knopp function (see [1] and references therein for a review). The issue was the study of the regularity.

Indeed, in 1918, Knopp [2] introduced a new family of nondifferentiable functions defined on the interval $[0,1]$. Going beyond the construction of Weierstrass of a continuous nondifferentiable function, his goal was to build examples of continuous functions for which one-sided limits of the difference quotient at all points do not exist. He considered the function $F_{a, b}$ given by the series, for $x \in[0,1]$,

$$
F_{a, b}(x)=\sum_{j=0}^{\infty} a^{j} \phi\left(b^{j} x\right),
$$

where $\phi(x)=\operatorname{dist}(x, \mathbb{Z}), 0<a<1$, and $b$ is an integer such that $a b>4$.
For $b=2$ and $a=2^{-\alpha}$, this function can be seen as a series expanded in the Faber-Schauder basis $\Lambda_{j, k}: x \mapsto 2^{j / 2} \Lambda\left(2^{j} x-\right.$ $k), j \in \mathbb{N}, k=0, \ldots, 2^{j}-1$, where $\Lambda$ is the Schauder function defined by $\Lambda(x)=\inf (x, 1-x)$ if $x \in[0,1]$ and 0 elsewhere. In fact

$$
F_{2^{-\alpha}, 2}(x)=\sum_{j=0}^{\infty} \sum_{k=0}^{j}-1
$$

We will write $F$ for $F_{2^{-\alpha}, 2}$ in the following.

Thus, for example, using the characterization of Lipschitz spaces with the help of coefficients in the Schauder-basis [3], one gets immediately the fact that $F$ belongs to $C^{\alpha}([0,1])$.

A further step to study the regularity of this function can be to follow the ideas developed in multifractal analysis. The goal in multifractal analysis is to study the sets of points where the function has a given pointwise regularity, and doing so to check if the regularity changes from point to point and quantify these changes. Recall the definition of Hölder pointwise regularity and local $L^{p}$ regularity. 
Definition 1. Let $x_{0} \in \mathbf{R}^{d}$ and $\alpha \geq 0$. A locally bounded function $f: \mathbf{R}^{d} \rightarrow \mathbf{R}$ belongs to $C^{\alpha}\left(x_{0}\right)$ if there exists $C>0$ and a polynomial $P=P_{x_{0}}$ with degree $\operatorname{deg}(P) \leq[\alpha]$ (integer part of $\alpha$ ), such that on a neighborhood of $x_{0}$,

$$
\left|f(x)-P_{x_{0}}(x)\right| \leq C\left|x-x_{0}\right|^{\alpha} .
$$

The pointwise Hölder exponent of $f$ at $x_{0}$ is $h_{f}\left(x_{0}\right)=\sup \{\alpha$ : $\left.f \in C^{\alpha}\left(x_{0}\right)\right\}$.

Definition 2 (see [4]). Let $x_{0} \in \mathbf{R}^{d}$. Let $p \in[1, \infty]$ and $u$ such that $u \geq-d / p$. Let $f$ be a function in $L_{\text {loc }}^{p}$. The function $f$ belongs to $T_{u}^{P}\left(x_{0}\right)$ if there exists $R>0$, a polynomial $P$ with $\operatorname{deg}(P) \leq u$, and $C>0$ such that

$$
\forall \rho \leq R:\left(\frac{1}{\rho^{d}} \int_{\left|x-x_{0}\right| \leq \rho}|f(x)-P(x)|^{p} d x\right)^{1 / p} \leq C \rho^{u} .
$$

The $p$-exponent of $f$ at $x_{0}$ is $u_{f}^{p}\left(x_{0}\right)=\sup \{u: f \in$ $\left.T_{u}^{p}\left(x_{0}\right)\right\}$.

Then again with the help of the Faber-Schauder basis one can prove that, for all $x_{0} \in[0,1], F$ is in $C^{\alpha}\left(x_{0}\right)$ (details for this technique can be found in [5]). It is then easy to check that actually $u_{p}^{F}\left(x_{0}\right)=h_{F}\left(x_{0}\right)=\alpha$ at all $x_{0} \in[0,1]$. Thus, from the point of view of various notions of regularity, even if it is not differentiable, the function $F$ is rather "regular" since one can compute at each point $x_{0}$ the same regularity exponent. This remark was actually the starting point of this work.

Indeed obviously the graph of the function has a very irregular behavior, and it has also some self-similarity properties. What can we say on the domain $\Omega=\{X=(x, y): y \leq$ $F(x)\}$ under the graph of $F$ ?

Denote in the following by $\rrbracket_{\Omega}$ the characteristic function of $\Omega$, which takes the value 1 on $\Omega$ and 0 outside $\Omega$.

A first reflex is to compute fractal dimensions of the boundary $\partial \Omega$. The box dimension of the graph can be derived by standard methods (see Tricot [6]) and is exactly $\operatorname{dim}_{B}(\partial \Omega)=2-\alpha$. Let us mention that Ciesielski $[7,8]$ proved results of this type for Schauder and Haar bases expansions in the case of more general families of functions. Jaffard [9] and Kamont and Wolnik [10] obtained then general formulas that allow deriving the box dimensions of the graphs of arbitrary functions from their wavelet expansions.

For what concerns the Hausdorff dimension of the graph of $F$, as far as we know, the question is not solved yet in its all generality. It was proved by Ledrappier [11] in 1992 to be $2-\alpha$ in the special case where $a=2^{\alpha-1}$ is an Erdös number. By the results of Solomyak [12] on Erdös numbers this amounts to have the computation for almost every $\alpha$ in $[0,1]$.

Beside the computation of the box and Hausdorff dimension, which provide global quantities to describe the graph of the function, several methods were recently developed to classify fractal boundaries with the help of pointwise exponents. The idea was to be able to give a finer description of the geometry of the boundary, since the pointwise behavior was studied. In [13], Jaffard and Mélot focused on the computation of the dimension of the set of points where $\rrbracket_{\Omega}$ has a given $p$-exponent in the sense of Definition 2. In [14], Heurteaux and Jaffard studied pointwise exponents more related to the geometry. These are the exponents we are actually interested in.

Indeed denote by meas the Lebesgue measure in $\mathbf{R}^{d}$ and $B(X, r)$ the $d$ dimensional open ball of center $X$ and radius $r>$ 0 . Heurteaux and Jaffard [14] gave the following definitions.

Definition 3. Let $\Omega$ be a domain of $\mathbf{R}^{d}$ and let $X_{0} \in \partial \Omega$. The point $X_{0}$ is weak $\alpha$-accessible in $\Omega$ if there exist $C>0$ and $r_{0}>0$ such that

$$
\forall r \leq r_{0} \quad \operatorname{meas}\left(\Omega \bigcap B\left(X_{0}, r\right)\right) \leq C r^{\alpha+d} .
$$

The supremum of all the values of $\alpha$ such that (5) holds is called the weak accessibility exponent in $\Omega$ at $X_{0}$. We denote it by $E_{\Omega}^{w}\left(X_{0}\right)$.

Example 4. Let $0<\beta<1$ and $\Omega=\left\{X=(x, y) \in \mathbf{R}^{2}:|y| \leq\right.$ $\left.|x|^{\beta}\right\}$. Denote by $\Omega^{c}$ the complement of $\Omega$. Then one can easily check that at each point $X_{1} \neq(0,0)$ of the boundary $\partial \Omega$ we have $E_{\Omega}^{w}\left(X_{1}\right)=0=E_{\Omega^{c}}^{w}\left(X_{1}\right)$ and at $X_{0}=(0,0)$ we have $E_{\Omega^{c}}^{w}\left(X_{0}\right)=(1 / \beta)-1$ and $E_{\Omega}^{w}\left(X_{0}\right)=0$.

Definition 5. Let $\Omega$ be a domain of $\mathbf{R}^{d}$ and let $X_{0} \in \partial \Omega$. The point $X_{0}$ is strong $\alpha$-accessible in $\Omega$ if there exist $C>0$ and $r_{0}>0$ such that

$$
\forall r \leq r_{0} \quad \operatorname{meas}\left(\Omega \bigcap B\left(X_{0}, r\right)\right) \geq C r^{\alpha+d}
$$

The infimum of all the values of $\alpha$ such that (6) holds is called the strong accessibility exponent in $\Omega$ at $X_{0}$. We denote it by $E_{\Omega}^{\mathcal{S}}\left(X_{0}\right)$.

The following proposition is given in [14].

Proposition 6. Let $X_{0} \in \partial \Omega$. Then

$$
\begin{aligned}
& d+E_{\Omega}^{w}\left(X_{0}\right)=\liminf _{r \rightarrow 0} \frac{\log \left(\text { meas }\left(\Omega \cap B\left(X_{0}, r\right)\right)\right)}{\log r}, \\
& d+E_{\Omega}^{s}\left(X_{0}\right)=\limsup _{r \rightarrow 0} \frac{\log \left(\operatorname{meas}\left(\Omega \cap B\left(X_{0}, r\right)\right)\right)}{\log r} .
\end{aligned}
$$

Obviously $E_{\Omega}^{s}\left(X_{0}\right) \geq E_{\Omega}^{w}\left(X_{0}\right)$. We will see that thanks to our result one can prove that these two exponents can be different.

Tricot [15] proved that these exponents are related to local dimension computation. Let us mention, without giving too much details, the relationship of this work [15] with these exponents. Indeed the author focuses on the formula

$$
H^{\phi}(E)=\liminf _{\varepsilon \rightarrow 0}\left\{\sum_{i \geq 0} \phi\left(E_{i}\right): E \subset \bigcup_{i \geq 0} E_{i}, \operatorname{diam}\left(E_{i}\right) \leq \varepsilon\right\}
$$

with $\phi: \mathbb{B}_{E} \rightarrow(0, \infty)$ some "set function" and $\mathbb{B}_{E}$ the set of closed balls centered on $E$. 

of

Given an open set $V$ such that $E \subset \partial V$ the special choice

$$
\phi_{\alpha}(B)=\frac{\operatorname{Vol}(B \cap V)}{\operatorname{Vol}(B)} \operatorname{diam}(B)^{\alpha}
$$

leads to definitions of Hausdorff, exterior and interior dimensions, and Packing, exterior and interior dimensions.

The following characterization, written for the setting we are interested in, holds.

Theorem 7 (see [15]). Let $\Omega$ be a bounded open set in $\mathbf{R}^{d}$ with boundary $\partial \Omega$ such that meas $(\partial \Omega)=0$. Let $X_{0} \in \Omega$. Let $r>0$ and

$$
\alpha\left(B\left(X_{0}, r\right)\right)=d-\frac{\log \left(\text { meas }\left(\Omega \bigcap B\left(X_{0}, r\right)\right)\right)}{\log (r)} .
$$

Then

$$
\begin{aligned}
& \liminf _{r \rightarrow 0} \alpha\left(B\left(X_{0}, r\right)\right)=\operatorname{dim}_{\text {int }}\left(\left\{X_{0}\right\}\right), \\
& \limsup _{r \rightarrow 0} \alpha\left(B\left(X_{0}, r\right)\right)=\operatorname{Dim}_{\text {int }}\left(\left\{X_{0}\right\}\right)
\end{aligned}
$$

with $\operatorname{dim}_{\text {int }}$ the Hausdorff interior dimension and $\mathrm{Dim}_{\text {int }}$ the Packing Hausdorff dimension.

We clearly have $\operatorname{dim}_{\text {int }}\left(\left\{X_{0}\right\}\right)=-E_{\Omega}^{w}\left(X_{0}\right)$ and $\operatorname{Dim}_{\text {int }}\left(\left\{X_{0}\right\}\right)=-E_{\Omega}^{s}\left(X_{0}\right)$. Let us stress that in the setting of Tricot $\operatorname{dim}_{\text {ext }}\left(\left\{X_{0}\right\}\right)=-E_{\Omega^{c}}^{w}\left(X_{0}\right)$ and $\operatorname{Dim}_{\text {ext }}\left(\left\{X_{0}\right\}\right)=$ $-E_{\Omega^{c}}^{s}\left(X_{0}\right)$ with $\Omega^{c}$ the complementary of $\Omega$ in $\mathbf{R}^{d}$. We rather refer to [15] for more details on local dimensions in their all generality.

We will compute these quantities at the points of the boundary $\partial \Omega$ of $\Omega=\left\{(x, y) \in \mathbf{R}^{2}: 0 \leq x \leq 1,0 \leq y \leq F(x)\right\}$, where $F$ is the function defined by (2). For that we will use the characterization of the maxima and minima done in [16]. This will yield the $p$-exponent at each point of $\rrbracket_{\Omega}$. We will actually derive the fact that the set of local extrema of the function is fully characterized by the set of points where this $p$-exponent has a given value.

We will also prove that the weak and strong accessibility exponents in $\Omega$ and $\Omega^{c}$ change from point to point on the graph $\partial \Omega$ of $F$. They also help to provide exact characterization of the sets of local maxima and local minima. Finally we will prove that there is a set of nontrivial Hausdorff dimensions such that the strong accessibility exponents in $\Omega$ and $\Omega^{c}$ are the same and the weak and strong accessibility exponents are different.

Let us emphasize that this is to our knowledge the first time that the computation of these exponents was done in a nearly exhaustive study on a given example. The characterization we get for the set of extrema raise several questions: is it a general property? Do other functions share it? Could it lead to a finer classification of functions in Hölder classes? We would like to address them in future works.

Let us come back now to our work. The outline of the paper is the following. In Section 2 we set our main result. In Section 3 some notations, preliminary remarks and technical lemmas, help us to prepare Section 4 where are the main proofs.

\section{Main Results}

2.1. Statement of Our Main Result. Our goal is to prove the following Theorem.

Theorem 8 (the main theorem). Let $a=2^{-\alpha}$ with $0<\alpha<1$ and $b=2$. Let $F$ be the function defined by (2).

Let $\Omega=\left\{X_{0}=(x, y) \in \mathbf{R}^{2}: 0 \leq x \leq 1,0 \leq y \leq F(x)\right\}$ and let $f=\mathbb{\square}_{\Omega}$.

Then, at each point $X_{0}$ of $\partial \Omega$, the graph of $F$, one has

(1) $u_{f}^{p}\left(X_{0}\right)=(1 / p)((1 / \alpha)-1)$ if and only if $F\left(X_{0}\right)$ is a local extremum of $F$. Furthermore

(a) $E_{\Omega}^{w}\left(X_{0}\right)=E_{\Omega}^{s}\left(X_{0}\right)=(1 / \alpha)-1$ if and only if $F\left(X_{0}\right)$ is a local maximum of $F$. And in this case $E_{\Omega^{c}}^{w}\left(X_{0}\right)=E_{\Omega^{c}}^{s}\left(X_{0}\right)=0$.

(b) $E_{\Omega^{c}}^{w}\left(X_{0}\right)=E_{\Omega^{c}}^{s}\left(X_{0}\right)=(1 / \alpha)-1$ if and only if $F\left(X_{0}\right)$ is a local minimum of $F$. And in this case $E_{\Omega}^{w}\left(X_{0}\right)=E_{\Omega}^{s}\left(X_{0}\right)=0$.

(2) In the other cases where $F\left(X_{0}\right)$ is not a local extremum of $F$, one has $E_{\Omega}^{w}\left(X_{0}\right)=E_{\Omega^{c}}^{w}\left(X_{0}\right)=0$.

(3) Furthermore one can find a subset $D_{\alpha} \subset \partial \Omega$ such that $E_{\Omega}^{\mathcal{S}}\left(X_{0}\right)=E_{\Omega^{c}}^{\mathcal{s}}\left(X_{0}\right)=(1 / \alpha)-1$ and $E_{\Omega}^{w}\left(X_{0}\right)=$ $E_{\Omega^{c}}^{w}\left(X_{0}\right)=0$ for all $X_{0} \in D_{\alpha}$. The orthogonal projection of $D_{\alpha}$ on $[0,1]$ has the Hausdorff dimension $\alpha$.

\section{Useful Notations and Results}

3.1. Lemmas for Practical Computation of the Exponents. From the computation of the weak accessibility exponent in $\Omega$ and $\Omega^{c}$ it is easy to derive the $p$-exponent. In [13], Jaffard and Mélot proved that $\rrbracket_{\Omega} \in T_{\alpha / p}^{p}\left(X_{0}\right)$ if and only if either $X_{0}$ is weak $\alpha$-accessible in $\Omega$ or $X_{0}$ is weak $\alpha$-accessible in $\Omega^{c}$. As a consequence we have

$$
p u_{\rrbracket_{\Omega}}^{p}\left(X_{0}\right)=\max \left(E_{\Omega}^{w}\left(X_{0}\right), E_{\Omega^{c}}^{w}\left(X_{0}\right)\right) .
$$

We will also need the following lemma.

Lemma 9. Let $f: \mathbf{R} \rightarrow \mathbf{R}$ be in $C^{\alpha}\left(x_{0}\right)$ with $0<\alpha<1$ and $\Omega$ the domain below (resp., above) the graph of $f$. Consider $X_{0}=\left(x_{0}, f\left(x_{0}\right)\right)$. Then $X_{0}$ is strong $(1 / \alpha)-1$, accessible in both $\Omega$ and $\Omega^{c}$.

Proof. Suppose that $\Omega$ is the domain below the graph of $f$. Without any loss of generality, we can assume that $X_{0}=(0,0)$. Let $r>0$. Since $f$ is in $C^{\alpha}(0)$ and $0<\alpha<1$ then there exists a constant $C \geq 0$ such that in neighborhood of 0

$$
|f(x)| \leq C|x|^{\alpha} .
$$

Thus

$$
-C|x|^{\alpha} \leq f(x) \leq C|x|^{\alpha} .
$$

Obviously meas $\left(\Omega^{c} \bigcap B\left(X_{0}, r\right)\right)$ (resp., meas $\left(\Omega \bigcap B\left(X_{0}, r\right)\right)$ ) is greater than the area $\mathscr{A}=C^{\prime} \int_{0}^{r} y^{1 / \alpha} d y=C^{\prime \prime} r^{1+1 / \alpha}$ above 
(resp., below) the graph of $x \mapsto C|x|^{\alpha}$ and below (resp., above) the square of side $r$ and center $X_{0}$.

The same results hold if $\Omega$ is the domain above the graph of $f$ (we have just to replace $\Omega$ by $\Omega^{c}$ ).

One of our goals for the points which are not extrema of $F$ will be to find sequences of local maxima or minima such that the following key lemma holds.

Lemma 10. Let $f: \mathbf{R} \rightarrow \mathbf{R}$ be in $C^{\alpha}(\mathbf{R})$ and $\Omega$ the domain below the graph of $f$. Consider $X_{0}=\left(x_{0}, f\left(x_{0}\right)\right)$. Suppose that there exists $c_{\alpha}>0$, a sequence $r_{n}$ of positive numbers, such that $r_{n} \rightarrow 0$ as $\left.n \rightarrow+\infty, x_{n} \in\right] x_{0}-r_{n}, x_{0}+r_{n}\left[\right.$, and $n_{0} \in \mathbb{N}$, such that

$$
\forall n \geq n_{0} \quad f\left(x_{n}\right)=f\left(x_{0}\right)-c_{\alpha} r_{n}^{\alpha} .
$$

Then $E_{\Omega^{c}}^{w}\left(X_{0}\right)=0$.

Proof. We can suppose that $\left.x_{n} \in\right] x_{0}-r_{n}, x_{0}$ [ (the case $x_{n} \in$ ]$x_{0}, x_{0}+r_{n}$ [ is similar). Then by the mean value theorem we can find $\left.b_{n} \in\right] x_{n}, x_{0}$ [ such that $f\left(b_{n}\right)=f\left(x_{0}\right)-r_{n}$. Let $b_{n}^{\prime}=$ $\inf \left\{b_{n} \in\right] x_{n}, x_{0}\left[; f\left(b_{n}\right)=f\left(x_{0}\right)-r_{n}\right\}$. Since $f$ is continuous we get $f\left(b_{n}^{\prime}\right)=f\left(x_{0}\right)-r_{n}$ and $\left.b_{n}^{\prime} \in\right] x_{n}, x_{0}$ [. It follows from the definition of $b_{n}^{\prime}$ and the mean value theorem that

$$
\forall t \in] x_{n}, b_{n}^{\prime}\left[\quad f(t)<f\left(x_{0}\right)-r_{n} .\right.
$$

Thus

$$
] x_{n}, b_{n}^{\prime}\left[\times\left[f\left(x_{0}\right)-r_{n}, f\left(x_{0}\right)+r_{n}\right] \subset B\left(X_{0}, r_{n}\right) \bigcap \Omega^{c} .\right.
$$

Therefore

$$
\operatorname{meas}\left(B\left(X_{0}, r_{n}\right) \bigcap \Omega^{c}\right) \geq 2\left|b_{n}^{\prime}-x_{n}\right| r_{n} .
$$

Since

$$
\begin{aligned}
f\left(x_{n}\right)-f\left(b_{n}^{\prime}\right) & =f\left(x_{0}\right)-c_{\alpha} r_{n}^{\alpha}-\left(f\left(x_{0}\right)-r_{n}\right) \\
& =r_{n}-c_{\alpha} r_{n}^{\alpha} \\
& \simeq-r_{n}^{\alpha},
\end{aligned}
$$

in the sense that there exists a constant $C \geq 1$ such that for every $n$ we have $(1 / C) r_{n}^{\alpha} \leq c_{\alpha} r_{n}^{\alpha}-r_{n} \leq C r_{n}^{\alpha}$.

Since $f$ belongs to $C^{\alpha}(\mathbf{R})$ we get

$$
C\left|b_{n}^{\prime}-x_{n}\right|^{\alpha} \geq\left|f\left(b_{n}^{\prime}\right)-f\left(x_{n}\right)\right| \simeq r_{n}^{\alpha} .
$$

Thus

$$
\left|b_{n}^{\prime}-x_{n}\right| \geq C^{\prime} r_{n} .
$$

Following (18) and (21) we get

$$
\text { meas }\left(B\left(X_{0}, r_{n}\right) \bigcap \Omega^{c}\right) \geq C r_{n}^{2} .
$$

Since meas $\left(B\left(X_{0}, r\right) \bigcap \Omega^{c}\right) \leq \operatorname{meas}\left(B\left(X_{0}, r\right)\right) \leq r^{2}$ for all $r \geq$ 0 we get

$$
\lim _{n \rightarrow+\infty} \frac{\log \left(\text { meas }\left(B\left(X_{0}, r_{n}\right) \bigcap \Omega^{c}\right)\right)}{\log \left(r_{n}\right)}=2 .
$$

Thus thanks to Proposition 6 we have $E_{\Omega^{c}}^{w}\left(X_{0}\right) \leq 0$ which yields $E_{\Omega^{c}}^{w}\left(X_{0}\right)=0$.

By replacing $f$ by $-f$ we also have the following result.

Lemma 11. Let $f: \mathbf{R} \rightarrow \mathbf{R}$ be in $C^{\alpha}(\mathbf{R})$ and $\Omega$ the domain below the graph of $f$. Consider $X_{0}=\left(x_{0}, f\left(x_{0}\right)\right)$. Suppose that there exist $c_{\alpha}>0, r_{n} \rightarrow 0$ as $\left.n \rightarrow+\infty, x_{n} \in\right] x_{0}-r_{n}, x_{0}+r_{n}[$, and $n_{0} \in \mathbb{N}$, such that

$$
\forall n \geq n_{0} \quad f\left(x_{n}\right)=f\left(x_{0}\right)+c_{\alpha} r_{n}^{\alpha} .
$$

Then $E_{\Omega}^{w}\left(X_{0}\right)=0$.

3.2. Dyadic Expansions and Approximation by Dyadics. We give some properties of the approximation of a point by the dyadics. Such properties will be used later.

Let $x \in[0,1]$. Set $i_{1}(x), \ldots, i_{j}(x), \ldots$, the binary digits of $x$; that is,

$$
x=\sum_{l=1}^{\infty} \frac{i_{l}(x)}{2^{l}} .
$$

(i) Note that dyadic points, that is, points $x=2^{-N} K$ with $K \in 2 \mathbb{N}+1$, are characterized by the fact that one can find $N>0$ such that $i_{N}(x)=1$ and $i_{n}(x)=0$ for $n>N$ or equivalently $i_{N}(x)=0$ and $i_{n}(x)=1$ for $n>N$.

Furthermore for $n>N$ the number $x-2^{-n}$ is dyadic. Since $2^{-n}=\sum_{j=n+1}^{\infty} 2^{-j}$, then $x-2^{-n}=\left(\sum_{j=1}^{N-1} i_{j} 2^{-j}\right)+$ $2^{-(N+1)}+2^{-(N+2)}+\cdots+2^{-n}$.

On the other hand $x+2^{-n}$ has the simple expansion $\sum_{j=1}^{N}\left(i_{j}(x) / 2^{j}\right)+\left(1 / 2^{n}\right)$.

We will denote by $\mathscr{D}$ the set of all dyadic points in $[0,1]$.

(ii) Let us come back to the general case with $x$ any point in $[0,1]$.

For each $j \in \mathbb{N}$, define $K_{j}\left(=K_{j}(x)\right)$ by

$$
\left|K_{j} 2^{-j}-x\right|=\inf _{k \in \mathbb{N}}\left|k 2^{-j}-x\right| .
$$

Set

$$
r_{j}(x)=\frac{\log \left|K_{j} 2^{-j}-x\right|}{\log 2^{-j}} .
$$

Define the rate of approximation of $x$ by dyadics as

$$
r(x)=\limsup _{j \mapsto \infty} r_{j}(x) .
$$

Since $\left|K_{j} 2^{-j}-x\right| \leq 2^{-j}$, then, for every $x$, we have $r(x) \geq 1$. If $x$ is dyadic then $r(x)=\infty$ (by taking the convention $\log 0=-\infty$ ). If $x$ is normal (i.e., the frequency of ones (or zeros) in the binary expansion of $x$ is equal to $1 / 2)$ then $r(x)=1$. 
(iii) If $r(x)>1$, following the definition of $r(x)$, then for any $\delta>0$ such that $r(x)-\delta>1$ one can find a subsequence $J_{n} \rightarrow+\infty$ for $n \rightarrow+\infty$ such that

$$
r_{J_{n}(x)} \leq 2^{-J_{n}(r(x)-\delta)}
$$

Let $J_{n}^{\prime}=\left[J_{n}(r(x)-\delta)\right]$. We then have

$$
K_{J_{n}} 2^{-J_{n}}-2^{-J_{n}^{\prime}} \leq x \leq K_{J_{n}} 2^{-J_{n}}+2^{-J_{n}^{\prime}}
$$

Thus, either $x$ belongs to the dyadic interval $\left[K_{J_{n}} 2^{-J_{n}}-\right.$ $\left.2^{-J_{n}^{\prime}}, K_{J_{n}} 2^{-J_{n}}\right]$ and in this case it satisfies $i_{J_{n}+1}(x)=$ $\cdots=i_{J_{n}^{\prime}-1}(x)=1$, or it belongs to the other interval $\left[K_{J_{n}} 2^{-J_{n}}, K_{J_{n}} 2^{-J_{n}}+2^{-J_{n}^{\prime}}\right]$ and in this case it satisfies $i_{J_{n}+1}(x)=\cdots=i_{J_{n}^{\prime}-1}(x)=0$.

In both cases let us notice that the binary expansion of $x$ contains chains of 0 or 1 whose length $J_{n}^{\prime}-J_{n} \sim J_{n}^{\prime}$ increases when $n \rightarrow+\infty$.

3.3. Approximation by Sequences of Maxima of F. We will see in the following that points in $[0,1]$ of the set

$$
\mathcal{S}=\left\{k \in \mathbb{N}, N_{0} \in \mathbb{N}, \frac{k}{2^{N_{0}}}+\frac{1}{3} \frac{1}{2^{N_{0}}}, \frac{k}{2^{N_{0}}}+\frac{2}{3} \frac{1}{2^{N_{0}}}\right\}
$$

will play a big role in this work, since they actually are the locations of the local maxima of the function $F$ (see below). Remark that they are characterized by the fact that, for each $x \in \mathcal{S}$, one can find $j_{0} \in \mathbb{N}$ such that for $j \geq j_{0}$ we have $i_{j}(x)+i_{j+1}(x)=1$.

As in the case of dyadic approximation we can define a rate of approximation by this kind of points.

Indeed let for $x \in[0,1]$

$$
\left|m_{j}-x\right|=\inf _{k \in \mathbb{N}}\left\{\left|\frac{k}{2^{j}}+\frac{1}{3} \frac{1}{2^{j}}-x\right|,\left|\frac{k}{2^{j}}+\frac{2}{3} \frac{1}{2^{j}}-x\right|\right\} .
$$

Define

$$
s_{j}(x)=\frac{\log \left|m_{j}-x\right|}{\log 2^{-j}} .
$$

Then the rate of approximation of $x$ by elements of $\mathcal{S}$ is given by

$$
s(x)=\limsup _{j \mapsto \infty} s_{j}(x) .
$$

Since $\left|m_{j}-x\right| \leq 2^{-j}$, then, for every $x$, we have $s(x) \geq 1$.

In the case of dyadic numbers, we have $s(x)=1$. But remark that in other nontrivial cases there is no obvious relationship between $s(x)$ and $r(x)$. Indeed one can check on the following examples that $s$ and $r$ can independently take any value.

(i) Let $x=\sum_{j=1}^{+\infty}\left(i_{j}(x) / 2^{j}\right)$ with $i_{3 k+1}(x)=0=i_{3 k+2}(x)$ and $i_{3 k+2}(x)=1$ for all $k \in \mathbb{N}$. Then we have $r(x)=$ $s(x)=1$. (ii) Let $u>1$. Then $x=\sum_{n=0}^{+\infty} 2^{-\left[u^{n}\right]}$ with $\left[u^{n}\right]$ the integer part of $u^{n}$. We have $r(x)=u$ whereas $s(x)=1$.

(iii) Let $u>1$. Then $x=\sum_{n=1}^{+\infty} 2^{-2 n}-\sum_{n=0}^{+\infty} 2^{-2\left[u^{n}\right]}$. We have $s(x)=u$ whereas $r(x)=1$.

(iv) Let $u>1$ and $s>1$. Let $x=\sum_{n=1}^{+\infty} 2^{-2\left[s^{n-1} u^{n}\right]}+$ $\sum_{k=\left[s^{n-1} u^{n}\right]+1}^{\left[s^{n} u^{n+1}\right]-1} 2^{-2 k}$. Then $r(x)=u$ and $s(x)=s$.

3.4. The Shift Operator. Since $0<\alpha<1$ it is easy to check that we obtain from (2) with $a=2^{-\alpha}$ and $b=2$

$F(x)$

$$
=\sum_{j=0}^{\infty} \sum_{i=\left(i_{1}, \ldots, i_{j}\right) \in\{0,1\}^{j}} 2^{-\alpha j} \Lambda\left(2^{j} x-2^{j-1} i_{1}-\cdots-2 i_{j-1}-i_{j}\right) .
$$

The term of (35) corresponding to $j=0$ is $\Lambda(x)$. But, the function $\Lambda$ is supported in $[0,1]$, and therefore $F$ vanishes outside $[0,1]$ and for $x \in[0,1]$

$$
F(x)=\sum_{j=0}^{\infty} 2^{-\alpha j} \Lambda\left(2^{j} x-2^{j-1} i_{1}(x)-\cdots-2 i_{j-1}(x)-i_{j}(x)\right) \text {. }
$$

For dyadic rationals $x, x=2^{-N} K$ with $K \in 2 \mathbb{N}+1$, as we already said it, there exist two binary expansions, one such that $i_{N}(x)=1$ and $i_{n}(x)=0$ for $n>N$ and another one such that $i_{N}(x)=0$ and $i_{n}(x)=1$ for $n>N$. The two righthand sides of (36) corresponding to the two choices of $i(x)$ give identical results.

Denote by $\tau$ the shift operator

$$
\tau x=\sum_{l=2}^{\infty} i_{l}(x) 2^{-l+1}=\sum_{l=1}^{\infty} i_{l+1}(x) 2^{-l} .
$$

Observe that

$$
\tau x= \begin{cases}2 x, & \text { if } x \in\left[0, \frac{1}{2}[,\right. \\ 2 x-1, & \text { if } x \in\left[\frac{1}{2}, 1\right] .\end{cases}
$$

Hence

$$
\begin{gathered}
F(x)=\sum_{j=0}^{\infty} 2^{-\alpha j} \Lambda\left(\tau^{j} x\right), \\
\tau^{j} x=\sum_{l=1}^{\infty} i_{l+j}(x) 2^{-l} .
\end{gathered}
$$

Our self-similar function is of the form $F(x)=$ $\sum_{j=0}^{\infty} \sum_{k=0}^{2^{j}-1} C_{j, k} \Lambda\left(2^{j} x-k\right)$ with

$$
\begin{gathered}
C_{j, k}=2^{-\alpha j} \quad \text { if } j \neq 0, k \neq 0, \\
C_{0,0}=1 .
\end{gathered}
$$


For $n \geq 1$, denote

$$
F_{n}(x)=\sum_{j=0}^{n} 2^{-\alpha j} \Lambda\left(\tau^{j} x\right)
$$

Remark that $F_{n}$ is affine on intervals of type $I_{n+1}=$ $\left[k / 2^{n+1},(k+1) / 2^{n+1}\right]$. Remark also that if $t \in[0,1]$, then $\Lambda(t)=(-1)^{i_{1}(t)} t+i_{1}(t)$. So, if $t^{\prime} \in[0,1]$ and $i_{1}(t)=i_{1}\left(t^{\prime}\right)$, then $\Lambda(t)-\Lambda\left(t^{\prime}\right)=(-1)^{i_{1}(t)}\left(t-t^{\prime}\right)$.

It follows that if $k / 2^{n+1}=\sum_{j=1}^{n+1}\left(i_{j} / 2^{j}\right)$ then the slope of $F_{n}$ at any point $x$ of the interval $] k / 2^{n+1},(k+1) / 2^{n+1}$ [ is exactly

$$
C_{n}=C_{n}(x)=\sum_{j=0}^{n}(-1)^{i_{j+1}(x)} 2^{(1-\alpha) j}=\sum_{j=0}^{n}(-1)^{i_{j+1}} 2^{(1-\alpha) j} .
$$

3.5. Extrema of $F$. We will need the following characterization of the extrema of $F$ proved in $[16,17]$. Let us start with the local and global minima.

Proposition 12. Let $0<\alpha<1$ and let $F$ be the function defined by (39); then

(i) 0 and 1 are the abscissas of the global minima of F;

(ii) the dyadic points are the abscissas of the minima of $F$ and furthermore

$$
\begin{aligned}
\min _{x \in I_{N}} F(x) & =\min \left[F_{N-1}\left(\frac{k}{2^{N}}\right), F_{N-1}\left(\frac{k+1}{2^{N}}\right)\right] \\
\text { with } I_{N} & =\left[k / 2^{N},(k+1) / 2^{N}\right] .
\end{aligned}
$$

In the case of the maxima, the statement of the result is slightly more technical. We need the following proposition of [17] using the same notations used previously.

Proposition 13. Let $0<\alpha<1$ and let $F$ be the function defined by (39). Let $t=2^{1-\alpha}$ and $X(p)$ the list of positions where $F(x)+p x$ attains its maximum on $[0,1]$. Let $\mathscr{M}(p)$ be the maximum on $[0,1]$ of $F(x)+p x$. Then

(i) $X\left(-\left(t^{N}-1\right) /(t-1)\right)=\left\{(1 / 3)\left(1 / 2^{N}\right),(2 / 3)\left(1 / 2^{N}\right)\right\}$ for $N=0,1, \ldots$

(ii) $X(p)=\left\{(1 / 3)\left(1 / 2^{N}\right)\right\}$ if $-\left(t^{N+1}-1\right) /(t-1)<p<$ $-\left(t^{N}-1\right) /(t-1)$.

(iii) $X(p)=1-X(-p)$ for all $p \neq 0$.

(iv) $\max _{x \in I_{N}} F(x)=F_{N-1}\left(k / 2^{N}\right)+2^{-N \alpha} \mathscr{M}\left(C_{N-1}((2 k+\right.$ 1) $\left.\left./ 2^{N+1}\right) / t^{N}\right)$.

The following proposition is a consequence of the previous one.

Proposition 14. Let $0<\alpha<1$ and let $F$ be the function defined by (39). Then

(i) $1 / 3$ and $2 / 3$ are the abscissas of the global maxima of $F$;

(ii) the abscissas of the local maxima of $F$ are the points of $\mathcal{S}$.
3.6. Approximation of Slopes of $F_{n}$. Suppose first we have some information about the dyadic expansion of $x$. Then we have the following lemma, which helps to control the behavior of the slopes of the affine function $F_{n-1}$.

Lemma 15. (1) Let $x=K / 2^{N}$ be a dyadic number. Then one can find $N_{0}>N, A>0$, and $B>0$ depending only on $x$ such that if $n \geq N_{0}$ then

$$
\begin{aligned}
& \forall y \in] x, x+2^{-n}\left[\quad A 2^{(1-\alpha) n} \leq C_{n-1}(y) \leq B 2^{(1-\alpha) n},\right. \\
& \forall y \in] x-2^{-n}, x\left[\quad-A 2^{(1-\alpha) n} \geq C_{n-1}(y) \geq-B 2^{(1-\alpha) n} .\right.
\end{aligned}
$$

(2) Let $x$ be the abscissa of a local maximum of $F$. Then one can find $J_{0}>0, A>0$, and $B>0$ such that for $n \geq J_{0}$

$$
\begin{gathered}
i_{n-1}(x)+i_{n}(x)=1, \\
C_{n-1}(x) C_{n}(x)<0, \\
A 2^{(1-\alpha) n \leq}(-1)^{i_{n+1}(x)} C_{n-1}(x) \leq B 2^{(1-\alpha) n} .
\end{gathered}
$$

(3) Let $x$ be a nondyadic point such that $r(x)>1$. Then one can find two subsequences $J_{n}$ and $J_{n}^{\prime}$ with $J_{n}^{\prime} / J_{n}>1$ for all $n$, such that $i_{J_{n}}(x)=i_{J_{n}^{\prime}+1}(x)$ and $i_{j}(x)+i_{J_{n}}(x)=1$ for $J_{n}<j<J_{n}^{\prime}+1$. Furthermore one can find $J_{0}>0, A>0$, and $B>0$ such that for $n>J_{0}$

$$
A 2^{(1-\alpha) J_{n}^{\prime}} \leq(-1)^{i_{J_{n+1}+1}(x)} C_{J_{n}^{\prime}-1}(x) \leq B 2^{(1-\alpha) J_{n}^{\prime}} .
$$

(4) Let $x$ be a nondyadic point such that $s(x)>1$. Then one can find two subsequences $J_{n}$ and $J_{n}^{\prime}$ with $J_{n}^{\prime} / J_{n}>1$ for all $n$, such that $i_{j}(x)+i_{j+1}(x)=1$ for $J_{n}<j<J_{n}^{\prime}$ and $i_{J_{n}^{\prime}}(x)=$ $i_{J_{n}^{\prime}+1}(x)$. Furthermore one can find $J_{0}>0, A>0$, and $B>0$ such that for $n>J_{0}$

$$
A 2^{(1-\alpha) J_{n}^{\prime}} \leq(-1)^{i_{J_{n}^{\prime}}^{\prime}(x)} C_{J_{n}^{\prime}-1}(x) \leq B 2^{(1-\alpha) J_{n}^{\prime}} .
$$

Proof. Consider the following cases.

Case 1 . The idea is very simple since it is a direct computation. Indeed following (43) we have for $y \in] x, x+2^{-n}[$

$$
\begin{aligned}
C_{n-1}(y)= & \sum_{j=0}^{n-1}(-1)^{i_{j+1}(y)} 2^{-\alpha j} 2^{j} \\
= & \sum_{j=0}^{N-2}(-1)^{i_{j+1}(y)} 2^{j(1-\alpha)}-2^{(N-1)(1-\alpha)}+\sum_{j=N}^{n-1} 2^{j(1-\alpha)} \\
= & \sum_{j=0}^{N-2}(-1)^{i_{j+1}(y)} 2^{j(1-\alpha)}+2^{N(1-\alpha)}\left(1-2^{\alpha-1}\right) \\
& \quad+2^{(N+1)(1-\alpha)} \frac{2^{(n-N-1)(1-\alpha)}-1}{2^{(1-\alpha)}-1} .
\end{aligned}
$$

The second equation with $y \in] x-2^{-n}, x$ [ can be computed in the same way, up to a change of signs. 
Thus one can find $N_{0}>N, A>0$, and $B>0$ such that (46) holds for $n>N_{0}$.

Case 2. It is enough to remark that $x$ has the following binary expansion:

$$
x=\frac{k_{N_{0}}}{2^{N_{0}}}+\sum_{l=0}^{\infty} \frac{1}{2^{2 l+1+N_{0}}} .
$$

As a consequence of Proposition 14, the same kind of computation yields Case 2 .

Case 3. Since $r(x)>1$, for any $\delta>0$ one can find two subsequences $J_{n}$ and $J_{n}^{\prime}$ such that $i_{J_{n}+1}(x)=\cdots=i_{J_{n}^{\prime}-1}(x)$ and $\left|x-K_{J_{n}} 2^{-J_{n}}\right| \leq 2^{-J_{n}^{\prime}}$ with $J_{n}^{\prime}=\left[(r(x)-\delta) J_{n}\right]$.

Suppose first eventually up to a small change of definition of $J_{n}^{\prime}$ that $i_{J_{n}}(x)=1=i_{J_{n}^{\prime}+1}(x)$ and $i_{J_{n}+1}(x)=\cdots=i_{J_{n}^{\prime}-1}(x)=$ $i_{J_{n}^{\prime}}(x)=0$.

Then with the same kind of computation as in Case 1 one gets

$$
A 2^{(1-\alpha) J_{n}^{\prime}} \leq C_{J_{n}^{\prime}-1}(x) \leq B 2^{(1-\alpha) J_{n}^{\prime}}
$$

In the other case $i_{J_{n}+1}(x)=\cdots=i_{J_{n}^{\prime}-1}(x)=i_{J_{n}^{\prime}}(x)=1$, the sign of the slope will be changed.

Case 4. This follows exactly the same ideas than previously. Since $s(x)>1$ for any $\delta>0$ one can find two subsequences $J_{n}$ and $J_{n}^{\prime}$ such that for all $J_{n}<j<J_{n}^{\prime} i_{j}(x)+i_{j+1}(x)=1$ and $i_{J_{n}}(x)=i_{J_{n}-1}(x), i_{J_{n}^{\prime}}(x)=i_{J_{n}^{\prime}+1}(x)$. Then with the same kind of computation as in Case 1 one gets

$$
A 2^{(1-\alpha) J_{n}^{\prime}} \leq(-1)^{i_{J_{n}^{\prime}}(x)} C_{J_{n}^{\prime}-1}(x) \leq B 2^{(1-\alpha) J_{n}^{\prime}} .
$$

Hence Lemma 15 holds.

If we do not have any further information on $x$, the following lemma will be useful.

Lemma 16. Let $x \in[0,1]$ be a nondyadic number.

(1) Then there exists $\delta>0$ and $\delta^{\prime}=1 /\left(2^{1-\alpha}-1\right)$ such that for all $n \in \mathbb{N}$ one can find $J_{n} \geq n$ such that

$$
\delta^{\prime} 2^{J_{n}(1-\alpha)}>\left|C_{J_{n}-1}(x)\right|>\delta 2^{J_{n}(1-\alpha)}
$$

(2) If $x \notin \mathcal{S}$ then there exists $\delta>0$ such that for all $n \geq 0$ there exists $J_{n} \geq n$ such that (54) holds and

$$
\begin{aligned}
& \text { (i) either }\left[C_{J_{n}-1}(x)>0 \text { and } i_{J_{n}+1}(x)=0\right] \text {, } \\
& \text { (ii) or }\left[C_{J_{n}-1}(x)<0 \text { and } i_{J_{n}+1}(x)=1\right] \text {. }
\end{aligned}
$$

Proof. (1) The upper bound is a straightforward computation. Suppose the contrary; that is, for all $\delta>0$ one can find $N \in \mathbb{N}$ such that for all $n \geq N$

$$
-\delta 2^{(1-\alpha) n} \leq C_{n-1}(x) \leq \delta 2^{(1-\alpha) n}
$$

If we suppose without loss of generality that $i_{n+1}(x)=0$ then at step $n$

$$
\begin{aligned}
&-\delta 2^{(1-\alpha) n} \leq C_{n-1}(x) \leq \delta 2^{(1-\alpha) n} \\
&-\delta 2^{(1-\alpha) n}+2^{(1-\alpha) n} \leq C_{n}(x) \leq \delta 2^{(1-\alpha) n}+2^{(1-\alpha) n} \\
&-\frac{\delta}{2^{1-\alpha}}+\frac{1}{2^{1-\alpha}} \leq \frac{C_{n}(x)}{2^{(1-\alpha)(n+1)}} \leq \frac{\delta}{2^{1-\alpha}}+\frac{1}{2^{1-\alpha}} .
\end{aligned}
$$

It is enough to choose $\delta$ such that $-\left(\delta / 2^{1-\alpha}\right)+\left(1 / 2^{1-\alpha}\right)>\delta$ to have a contradiction.

(2) Suppose the contrary; that is, there exists $x \notin \mathcal{S}$ and that, for all $\beta>0$, there exists $N \in \mathbb{N}$, such that for all $n \geq N$

(a) either $\left|C_{n-1}(x)\right| \leq \beta 2^{J_{n}(1-\alpha)}$,

(b) or $\left[C_{n-1}(x)<0\right.$ and $\left.i_{n+1}(x)=0\right]$,

(c) or $\left[C_{n-1}(x)>0\right.$ and $\left.i_{n+1}(x)=1\right]$.

Remark first that the points of $\mathcal{S}$ satisfy exactly (2b) and (2c). Indeed for $x \in \mathcal{S}$ and assuming that $i_{n_{0}+1}(x)=1, x$ has a binary expansion (51).

Thus following (43) the slope $C_{n-1}(x)$ satisfies

$$
\begin{gathered}
C_{n-1}(x)=\sum_{j=0}^{N_{0}-1}(-1)^{i_{j+1}(x)} 2^{(1-\alpha) j}+\sum_{j=N_{0}}^{n-1} 2^{(1-\alpha) j}(-1)^{i_{j+1}(x)} \\
=\sum_{j=0}^{N_{0}-1}(-1)^{i_{j+1}(x)} 2^{(1-\alpha) j} \\
\quad+2^{N_{0}(1-\alpha)} \frac{\left(-2^{(1-\alpha)}\right)^{n-N_{0}}-1}{1+2^{1-\alpha}} .
\end{gathered}
$$

Hence, for $n$ being large enough (2b) and (2c) are satisfied.

Our goal is thus to prove that if we choose $\beta$ being small enough then only (2b) and (2c) can be satisfied, which will lead to the fact that $x \in \mathcal{S}$ and thus to a contradiction.

Let us start by the following special cases.

(i) We claim that if one can find $k$ being large enough such that $C_{k-1}(x)=0$ then $x \in \mathcal{S}$, which is a contradiction.

Let us prove this claim.

We will need the following sequence: let for $n \in \mathbb{N}^{\star}$

$$
\begin{aligned}
d_{n} & =2^{-(1-\alpha)} \sum_{j=0}^{n}(-1)^{j} 2^{-j(1-\alpha)} \\
& =\frac{2^{-(1-\alpha)}}{1+2^{-(1-\alpha)}}\left(1-(-1)^{n+1} 2^{-(n+1)(1-\alpha)}\right) .
\end{aligned}
$$

We clearly have $d_{n} \geq d_{1}>0$ for all $n \geq 1$.

Choose $\beta \leq d_{1} / 2$ and $N$ such that the hypotheses are satisfied.

Suppose that $k \geq N+1$ is such that $C_{k-1}(x)=0$. Then $\left|C_{k}(x)\right|=2^{k(1-\alpha)}=2^{-(1-\alpha)} 2^{(k+1)(1-\alpha)}$.

Remark that $\beta<d_{1} \leq 2^{-(1-\alpha)}$ and thus $\left|C_{k}(x)\right|>$ $\beta 2^{(k+1)(1-\alpha)}$. 
Suppose without loss of generality that $C_{k}(x)>0$ (the case $C_{k}(x)<0$ is symetrical and can be proved in exactly the same way). Thus $i_{k+2}(x)=1$ and

$$
\begin{aligned}
C_{k+1}(x) & =-2^{(k+1)(1-\alpha)}+2^{k(1-\alpha)} \\
& =-2^{(k+2)(1-\alpha)}\left(2^{-(1-\alpha)}-2^{-2(1-\alpha)}\right) \\
& <-\beta 2^{(k+2)(1-\alpha)} .
\end{aligned}
$$

Let us prove by induction on $n$ that for all $n \in \mathbb{N}^{\star}$

$$
\left|C_{k+n}(x)\right|=d_{n} 2^{(k+n+1)(1-\alpha)}, \quad(-1)^{n} C_{k+n}(x)>0 .
$$

We just proved that $(\mathrm{P})$ is true for $n=1$.

Suppose that for $n \in \mathbb{N}^{\star}(\mathrm{P})$ is true. Suppose without loss of generality that $C_{k+n}(x)>0$ (the case $C_{k+n}(x)<0$ is symetrical and can be proved in exactly the same way). Thus $i_{k+n+2}(x)=1$ and

$$
\begin{aligned}
C_{k+n+1}(x) & =C_{k+n}(x)-2^{(n+k+1)(1-\alpha)} \\
& =d_{n} 2^{(k+n+1)(1-\alpha)}-2^{(n+k+1)(1-\alpha)} \\
& =-\left(-2^{-(1-\alpha)} d_{n}+2^{-(1-\alpha)}\right) 2^{(k+n+2)(1-\alpha)} .
\end{aligned}
$$

Since $d_{n}$ satisfies exactly $d_{n+1}=-2^{-(1-\alpha)} d_{n}+2^{-(1-\alpha)}$, we have the result and property $(\mathrm{P})$ is satisfied at level $n+1$.

Thus for all $n \in \mathbb{N}^{\star}(\mathrm{P})$ is true. Recall that since $\beta<d_{1} \leq$ $d_{n}$ for all $n \in \mathbb{N}^{*}$, this implies that for all $n \in \mathbb{N}^{*} i_{k+n+2}(x)+$ $i_{k+n+3}(x)=1$, which is exactly the characterization of the points in $\mathcal{S}$ and is in contradiction with the hypothesis $x \notin \mathcal{S}$.

In the following we will always keep the hypothesis $0<$ $\beta \leq d_{1} / 2$ so that for $n$ large enough we always have $C_{n}(x) \neq 0$.

(ii) We now consider the case where $\left|C_{n-1}(x)\right|$ is close to the value of $\beta 2^{n(1-\alpha)}$ and prove that this yields that $x \in \mathcal{S}$, thus a contradiction.

Let $0<\beta \leq d_{1} / 2$ and $\beta^{\prime}>0$ whose value will be precised later on.

Suppose $n \geq N$ is such that $\beta^{\prime} 2^{n(1-\alpha)}>C_{n-1}(x)>\beta 2^{n(1-\alpha)}$ (the case $C_{n-1}(x)<-\beta 2^{n(1-\alpha)}$ can be done exactly in the same way). Then $i_{n+1}(x)=1$ and $C_{n}(x)=C_{n-1}(x)-2^{n(1-\alpha)}$; hence

$$
\begin{aligned}
\beta 2^{n(1-\alpha)}-2^{n(1-\alpha)} \leq & C_{n}(x) \leq \beta^{\prime} 2^{n(1-\alpha)}-2^{n(1-\alpha)} \\
(\beta-1) 2^{-(1-\alpha)} 2^{(n+1)(1-\alpha)} & \leq C_{n}(x) \\
& \leq\left(\beta^{\prime}-1\right) 2^{-(1-\alpha)} 2^{(n+1)(1-\alpha)} .
\end{aligned}
$$

Choose $\beta^{\prime}$ such that $\left(\beta^{\prime}-1\right) 2^{-(1-\alpha)}<-\beta$; hence $\beta<\beta^{\prime}<$ $(1-\beta) / 2^{-(1-\alpha)}$, which is possible since $\beta \leq d_{1} / 2<2^{-(1-\alpha)} /(1+$ $\left.2^{-(1-\alpha)}\right)$.

This yields $i_{n+2}(x)=0$. Thus

$$
\begin{aligned}
C_{n+1}(x) & =C_{n-1}(x)+\left(-2^{n(1-\alpha)}+2^{(n+1)(1-\alpha)}\right) \\
& >\left(2^{-(1-\alpha)}-2^{-2(1-\alpha)}\right) 2^{(n+2)(1-\alpha)} \\
& >\beta 2^{(n+2)(1-\alpha)} .
\end{aligned}
$$

Let us prove by induction on $k$ that, for all $k \in \mathbb{N}$,

$$
(-1)^{k+1} C_{n+k}(x)>\beta 2^{(n+k+1)(1-\alpha)} .
$$

We just prove that the case $k=0$ is true.

Suppose one can find $k \in \mathbb{N}$ such that $(\mathrm{Q})$ is true for all $0 \leq k^{\prime} \leq k$.

Let us prove that it is true at $k+1$. Without loss of generality suppose $C_{n+k}(x)>0$; thus $i_{n+k+2}(x)=1$.

We have

$$
\begin{aligned}
C_{n+k+1}(x) & =C_{n+k-1}(x)+2^{(n+k)(1-\alpha)}-2^{(n+k+1)(1-\alpha)} \\
& <-\left(2^{-(1-\alpha)}-2^{-2(1-\alpha)}\right) 2^{(n+k+2)(1-\alpha)} \\
& <-\beta 2^{(n+k+2)(1-\alpha)} .
\end{aligned}
$$

This proves that $(\mathrm{Q})$ is true at $k+1$.

Thus by induction (Q) is true for all $k \in \mathbb{N}$. This means that for all $k \in \mathbb{N} i_{n+k+2}(x)+i_{n+k+3}(x)=1$, and thus $x \in \mathcal{S}$, hence the contradiction.

(iii) We now study the case where $\left|C_{n-1}(x)\right| \leq \beta 2^{n(1-\alpha)}$ and prove that if we choose $\beta$ being small enough then it will lead to $x \in \mathcal{S}$.

Indeed let $\beta \leq \inf \left(d_{1} / 2, d_{1}^{\prime} / 2\right)$ with

$$
d_{1}^{\prime}=\frac{2^{-(1-\alpha)}-2^{-2(1-\alpha)}}{1+2^{-2(1-\alpha)}} .
$$

And suppose $n \geq N$ such that $\left|C_{n-1}(x)\right| \leq \beta 2^{n(1-\alpha)}$. Suppose $i_{n+1}(x)=0$ without lost of generality. Thus we have

$$
\begin{aligned}
& C_{n}(x)=C_{n-1}(x)+2^{n(1-\alpha)}, \\
&(-\beta+1) 2^{-(1-\alpha)} 2^{(n+1)(1-\alpha)} \leq C_{n}(x) \\
& \leq(\beta+1) 2^{-(1-\alpha)} 2^{(n+1)(1-\alpha)} .
\end{aligned}
$$

Remark that with the choice of $\beta$ we made, we have on one hand $\beta<(-\beta+1) 2^{-(1-\alpha)}$ and on the other hand $\beta<(\beta+$ $1) 2^{-(1-\alpha)}<(1-\beta) / 2^{-(1-\alpha)}$. Thus following the previous result using $\beta^{\prime}=(\beta+1) 2^{-(1-\alpha)}, x \in \mathcal{S}$ and we have a contradiction.

(iv) We consider the case where $C_{n-1}(x)>0$ and $C_{n}(x)<$ 0 for $n$ being large enough under the previous range of values of $\beta$.

Let $\beta \leq \inf \left(d_{1} / 2, d_{1}^{\prime} / 2\right)$ (recall that $d_{1}$ is defined by (58) and $d_{1}^{\prime}$ by (64)).

And suppose that for $n \geq N$ we have $C_{n-1}(x)>0$ and $C_{n}(x)<0$.

Following the previous case we have $C_{n-1}(x)>\beta 2^{n(1-\alpha)}$ and $C_{n}(x)<-\beta 2^{(n+1)(1-\alpha)}$. Thus $i_{n+1}(x)=1$ and $i_{n+2}(x)=0$.

Then

$$
\begin{aligned}
C_{n+1}(x) & =C_{n-1}(x)-2^{n(1-\alpha)}+2^{(n+1)(1-\alpha)} \\
& >\beta 2^{(n+2)(1-\alpha)}
\end{aligned}
$$

since by definition of $\beta$ and $d_{1}$ we have $2^{-(1-\alpha)}-2^{-2(1-\alpha)} \geq$ $d_{1}>\beta$. Thus $i_{n+3}(x)=1$. 
We have $C_{n+2}(x)=C_{n}(x)+2^{(n+1)(1-\alpha)}-2^{(n+2)(1-\alpha)}<$ $-\beta 2^{(n+3)(1-\alpha)}$.

A proof by induction exactly in the same way as previously proved yields that for $k \geq 0$ we have $C_{n+2 k+1}(x)>0$ and $C_{n+2 k}(x)<0$; thus $i_{n+2 k+2}(x)+i_{n+2 k+3}(x)=1$ for all $k \in \mathbb{N}$ and we have $x \in \mathcal{S}$, hence a contradiction.

We will now go for the main proof, taking into account what we just proved.

In the following we will consider $\delta>0$ and $J_{n}$ defined as in Point $1, \beta=\inf \left(d_{1} / 2, \delta / 2, d_{1}^{\prime} / 2\right)$, and $n$ such that $J_{n} \geq N$. Thus for all $n \in \mathbb{N}\left|C_{I_{n-1}}(x)\right| / 2^{J_{n}(1-\alpha)}>\delta>\beta$.

Suppose $C_{J_{n}-1}(x)^{n}>0$. This means that $C_{J_{n}}(x)=$ $C_{J_{n}-1}(x)-2^{J_{n}(1-\alpha)}<C_{J_{n-1}}(x)$. The only case we want to consider is $C_{J_{n}}(x)>\beta 2^{\left(J_{n}+1\right)(1-\alpha)}$ since for all the other cases the previous points yield $x \in \mathcal{S}$.

Thus $i_{J_{n}+2}(x)=1$. It is clear that one can find $k \in \mathbb{N}$ such that for all $0 \leq k^{\prime} \leq k C_{J_{n}+k^{\prime}}(x)>\beta 2^{\left(J_{n}+k^{\prime}+1\right)(1-\alpha)}$ and $i_{J_{n}+k^{\prime}+2}(x)=1$ and $C_{J_{n}+k+1}(x)<\beta 2^{\left(J_{n}+k+2\right)(1-\alpha)}$.

Hence either $\left|C_{J_{n}+k+1}(x)\right| \leq \beta 2^{\left(J_{n}+k+2\right)(1-\alpha)}$ and $x \in$ $\mathcal{S}$ or $C_{J_{n}+k+1}(x)<-\beta 2^{\left(J_{n}+k+2\right)(1-\alpha)}$ and since $C_{J_{n}+k}(x)>$ $\beta 2^{\left(J_{n}+k+1\right)(1-\alpha)}$ we get the desired result.

In all cases we proved that Points (2a), (2b), and (2c) lead to $x \in \mathcal{S}$, which is a contradiction. Hence Lemma 16 holds.

\section{Computation of Weak and Strong Accessible Exponents}

4.1. Case of Dyadic Points. We will prove the following proposition.

Proposition 17. If $x$ is a dyadic point, and $X=(x, F(x))$ then

$$
\begin{gathered}
E_{\Omega^{c}}^{w}(X)=\frac{1}{\alpha}-1, \quad E_{\Omega}^{w}(X)=0, \\
E_{\Omega^{c}}^{s}(X)=\frac{1}{\alpha}-1, \quad E_{\Omega}^{s}(X)=0, \\
u_{f}^{p}(X)=\frac{1}{p}\left(\frac{1}{\alpha}-1\right) .
\end{gathered}
$$

If $x$ is a dyadic point, that is, $x=2^{-N} K$ with $K \in 2 \mathbb{N}+1$, we consider its binary expansion in which $i_{N}(x)=1$ and $i_{n}(x)=$ 0 for $n>N$. For $n>N$ the number $x-2^{-n}$ is dyadic. Since $2^{-n}=\sum_{j=n+1}^{\infty} 2^{-j}$ then $x-2^{-n}=\left(\sum_{j=1}^{N-1} i_{j} 2^{-j}\right)+2^{-(N+1)}+$ $2^{-(N+2)}+\cdots+2^{-n}$. On the other hand $x+2^{-n}$ has the simple expansion $\left(\sum_{j=1}^{N}\left(i_{j}(x) / 2^{j}\right)\right)+\left(1 / 2^{n}\right)$.

Remark that $F_{N-1}(x)=F_{n-1}(x)=F(x)$ for $n>N$ and $F_{n-1}\left(x+2^{-n}\right)=F\left(x+2^{-n}\right)$.

Any point $y$ in the interval ] $x, x+2^{-n}$ [ satisfies the expansion $i_{N}(y)=i_{N+1}(y)=\cdots=i_{n-1}(y)=i_{n}(y)=0$.

It follows that

$$
\begin{aligned}
F\left(x+2^{-n}\right)-F(x) & =F_{n-1}\left(x+2^{-n}\right)-F_{n-1}(x) \\
& =2^{-n} C_{n-1}(y)
\end{aligned}
$$

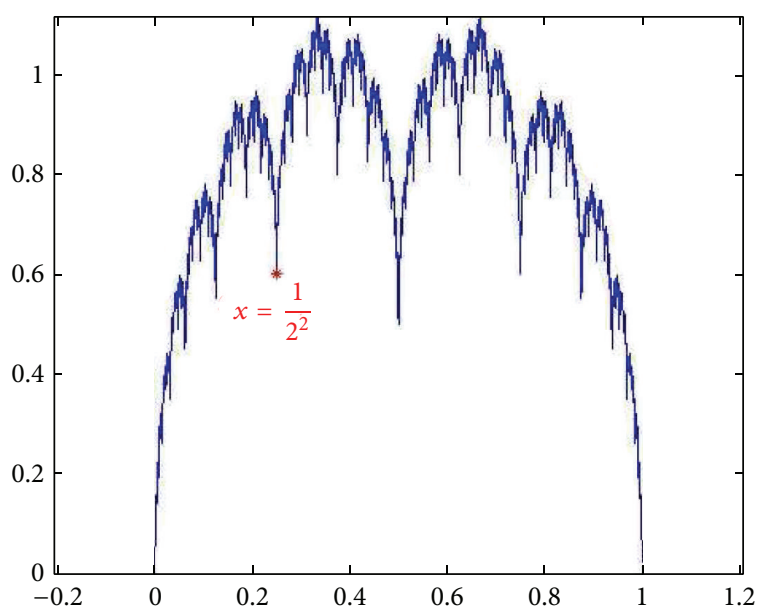

Figure 1: Overview of the function.

Following Lemma 15 and Case 1 there exist two constants $A>0$ and $B>0$ and $J_{0} \geq N$ (which depend only on the given dyadic point $x$ ) such that

$$
\forall n \geq J_{0} \quad A 2^{-\alpha n} \leq F\left(x+2^{-n}\right)-F(x) \leq B 2^{-\alpha n} .
$$

Thus we have $F\left(x+2^{-n}\right)-F(x) \geq A .2^{-\alpha n}$.

On the other hand, following remarks of Section 3.2, for any $y \in] x-2^{-n}, x\left[\right.$ we have $i_{N}(y)=1=\cdots=i_{n}(y)$. Thus

$$
\begin{aligned}
F\left(x-2^{-n}\right)-F(x) & =F_{n-1}\left(x-2^{-n}\right)-F_{n-1}(x) \\
& =-C_{n-1}(y) 2^{-n}
\end{aligned}
$$
$J_{0}$

Whence, following Lemma 15 and Case 1 we have for $n \geq$

$$
\forall n \geq J_{0} \quad A 2^{-\alpha n} \leq-F\left(x-2^{-n}\right)+F(x) \leq B 2^{-\alpha n} .
$$

Let $\rho>0$ and $J \geq J_{0}$ such that $2^{-J-1} \leq \rho \leq 2^{-J}$.

Since $F \geq F_{J}$, then $\Omega_{j} \subset \Omega$, where $\Omega_{J}$ is the domain below the graph of $F_{J}$. So

$$
\text { meas }\left(B(X, \rho) \cap \Omega^{c}\right) \leq \operatorname{meas}\left(B(X, \rho) \cap \Omega_{J}^{c}\right) .
$$

But meas $\left(B(X, \rho) \cap \Omega_{J}^{c}\right)$ is smaller than the area $h b / 2$ of a triangle with altitude $h$ issued from $X$ and a corresponding hypotenuse $b$ (see Figures 1 and 2 ).

Clearly, we can take $h \sim 2^{-J}$. On the other hand, if we write $2^{-(j+1)}<b / 2<2^{-j}$ with $j \geq J$, then, using properties (69) and (71) (in which we replace $n \alpha$ by $J$ ), we get $b / 2 \sim 2^{-J / \alpha}$. Since $\alpha<1,(69)$ and (71) are valid with $n=J / \alpha \geq J_{0}$.

Whence

$$
\operatorname{meas}\left(B(X, \rho) \cap \Omega^{c}\right) \leq C \rho^{1+(1 / \alpha)} .
$$

We conclude that

$$
E_{\Omega^{c}}^{w}(X) \geq \frac{1}{\alpha}-1
$$

with $y$ any of the points of the interval $] x, x+2^{-n}[$. 


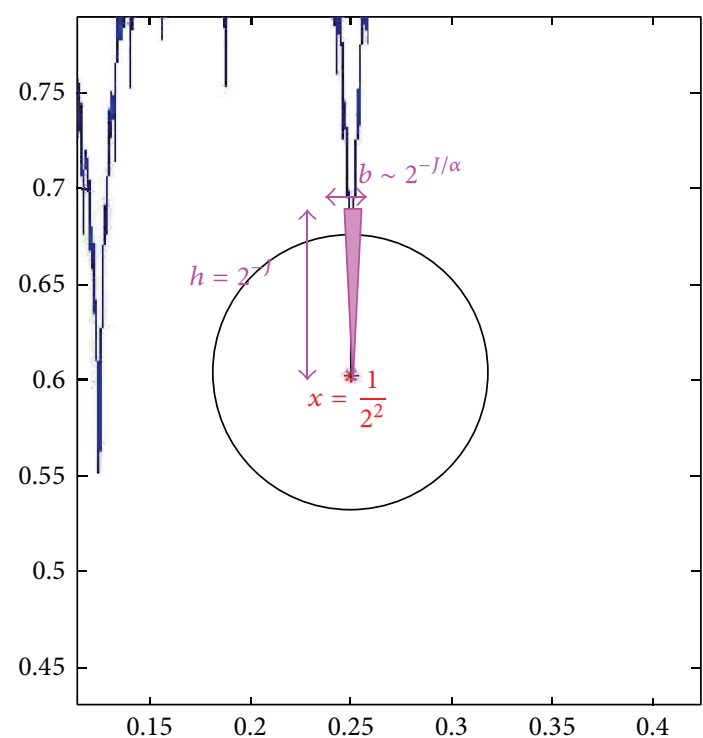

FIGURE 2: Zoom at the point $x=1 / 2^{2}$.

Since $E_{\Omega^{c}}^{w}(X) \leq E_{\Omega^{c}}^{s}(X) \leq(1 / \alpha)-1$ this yields

$$
E_{\Omega^{c}}^{s}(X)=\frac{1}{\alpha}-1 \text {. }
$$

Since $f=1_{\Omega}$ then

$$
u_{f}^{p}(X) \geq \frac{1}{p}\left(\frac{1}{\alpha}-1\right)
$$

Since $\operatorname{meas}(B(X, r))=\operatorname{meas}(B(X, r) \bigcap \Omega)+$ meas $\left(B(X, r) \bigcap \Omega^{c}\right)$ we get $E_{\Omega}^{\mathcal{s}}(X)=0$; hence $E_{\Omega}^{w}(X)=0$.

Whence Proposition 17 holds.

4.2. Case of a Local Maximum of F. We will prove the following proposition.

Proposition 18. Let $X=(x, F(x))$.

If $x$ is a local maximum,

$$
\begin{gathered}
E_{\Omega}^{w}(X)=\frac{1}{\alpha}-1, \quad E_{\Omega^{c}}^{w}(X)=0, \\
E_{\Omega}^{s}(X)=\frac{1}{\alpha}-1, \quad E_{\Omega^{c}}^{s}(X)=0, \\
u_{f}^{p}(X)=\frac{1}{p}\left(\frac{1}{\alpha}-1\right) .
\end{gathered}
$$

Let $x$ be a local maximum of $F$. There is an interval $I$ containing $x$ such that for all $x^{\prime} \in I, F(x) \geq F\left(x^{\prime}\right)$. Let $N$ be such that the dyadic interval $\left[k_{N} / 2^{N},\left(k_{N}+1\right) / 2^{N}\right]$ which contains $x$ is contained in $I$.

Following Proposition 14, we know that $x$ has the binary expansion (51); that is, $x=\left(k_{N_{0}} / 2^{N_{0}}\right)+\sum_{l=0}^{\infty}\left(1 / 2^{2 l+1+N_{0}}\right)$.

As a consequence of Lemma 15, and following Case (2), one can find $J_{0}$ and two constants $A$ and $B$ such that for $n \geq J_{0}$ (47) holds. Remark that it implies clearly that for $n \geq J_{0} i_{n}(x)=1$ if $n$ is odd, and $i_{n}(x)=0$ if $n$ is even.
Our goal now is to evaluate $F(x)-F\left(x^{\prime}\right)$ with $x^{\prime}$ in the interval $\left[k_{n} / 2^{n},\left(k_{n}+1\right) / 2^{n}\right] \subset\left[k_{N} / 2^{N}, k_{N+1} / 2^{N}\right]$ and $x^{\prime} \neq x$. If $x^{\prime}$ is a dyadic then we take its expansion of type $i_{j}\left(x^{\prime}\right)=0$ for $j$ to be large enough.

Let $m \geq n$ be the smallest integer such that $i_{m}(x)=$ $i_{m}\left(x^{\prime}\right)$ and $i_{m+1}(x) \neq i_{m+1}\left(x^{\prime}\right)$. To fix the ideas, suppose that $i_{m+1}(x)=1$ and $i_{m+1}\left(x^{\prime}\right)=0$. Thus

$\frac{1}{32^{m-1}} \geq x-x^{\prime} \geq \frac{1}{2^{m+1}}+\frac{1}{2^{m+3}}-\sum_{j=m+2}^{\infty} \frac{1}{2^{j}} \geq \frac{1}{2^{m+3}}$.

Since $i_{m}(x)=0$ we have $C_{m-1}(x)>0$.

Thus

$$
\begin{aligned}
F(x)-F\left(x^{\prime}\right)= & C_{m-1}(x)\left(x-x^{\prime}\right) \\
& +\sum_{k=m}^{+\infty} 2^{-k \alpha}\left(\Lambda\left(\tau^{k} x\right)-\Lambda\left(\tau^{k} x^{\prime}\right)\right) .
\end{aligned}
$$

We have

$$
\begin{aligned}
A 2^{m(1-\alpha)} 2^{-m-3} & =C_{1} 2^{-m \alpha} \\
& \leq C_{m-1}(x)\left(x-x^{\prime}\right) \\
& \leq \frac{B 2^{m(1-\alpha)} 2^{-m-1}}{3} \\
& =C_{2} 2^{-m \alpha} .
\end{aligned}
$$

Since for $k \geq m-1$ we have $\Lambda\left(\tau^{k} x\right)=1 / 3$, this yields

$$
0 \leq \sum_{k=m}^{+\infty} 2^{-k \alpha}\left(\Lambda\left(\tau^{k} x\right)-\Lambda\left(\tau^{k} x^{\prime}\right)\right) \leq C_{3} 2^{-m \alpha} .
$$

Thus we have

$$
C_{1} 2^{-m \alpha} \leq F(x)-F\left(x^{\prime}\right) \leq\left(C_{3}+C_{2}\right) 2^{-m \alpha} .
$$

Let us compute the weak and strong exponents at $x$. Let $\rho$ and $J \geq J_{0}$ such that $2^{-J-1} \leq \rho \leq 2^{-J}$. Thus obviously

$$
\operatorname{meas}(B(X, \rho) \bigcap \Omega) \leq \operatorname{meas}\left(B\left(X, 2^{-j}\right) \bigcap \Omega\right) \text {. }
$$

Remark first that if $\left(x^{\prime}, y^{\prime}\right) \in B\left(X, 2^{-J}\right) \bigcap \Omega$ then $\mid x-$ $x^{\prime}\left|<2^{-J},\right| y^{\prime}-F(x) \mid<2^{-J}$, and $y^{\prime} \leq F\left(x^{\prime}\right)$. Since $x$ is a local maximum on the interval $\left[k_{J} / 2^{J},\left(k_{J}+1\right) / 2^{J}\right]$, then $y^{\prime} \leq F\left(x^{\prime}\right) \leq F(x)$ and so $0 \leq F(x)-F\left(x^{\prime}\right)<2^{-J}$. Hence $\left(x^{\prime}, F\left(x^{\prime}\right)\right) \in B\left(X, 2^{-J}\right) \bigcap \Omega$.

Furthermore since $F\left(x^{\prime}\right)$ satisfies $0 \leq F(x)-F\left(x^{\prime}\right) \leq 2^{-J}$, and following (84) $x^{\prime}$ belongs to $\left[x-C 2^{-J / \alpha}, x+C 2^{-J / \alpha}\right]$ with $C$ depending only on $C_{3}+C_{2}$.

Thus $B\left(X, 2^{-J}\right) \cap \Omega$ is contained in a rectangle of length $2^{-J}$ and width $C 2^{-J / \alpha}$.

This yields

$\operatorname{meas}(B(X, \rho) \bigcap \Omega) \leq C 2^{-J(1+(1 / \alpha))} \leq C^{\prime} \rho^{1+(1 / \alpha)}$. 
We can conclude that

$$
E_{\Omega}^{w}(X) \geq \frac{1}{\alpha}-1 .
$$

Since $E_{\Omega}^{w}(X) \leq E_{\Omega}^{s}(X) \leq(1 / \alpha)-1$ this yields

$$
E_{\Omega}^{w}(X)=E_{\Omega}^{s}(X)=\frac{1}{\alpha}-1
$$

Since $\operatorname{meas}(B(X, \rho))=\operatorname{meas}(B(X, \rho) \bigcap \Omega)+\operatorname{meas}(B(X$, $\rho) \bigcap \Omega^{c}$ ) we get

$$
E_{\Omega^{c}}^{\mathcal{S}}(X)=E_{\Omega^{c}}^{w}(X)=0 .
$$

And finally

$$
u_{f}^{p}(X)=\frac{1}{p}\left(\frac{1}{\alpha}-1\right) .
$$

4.3. Case of $x \notin \mathscr{D} \bigcup \mathcal{S}$. If $x \notin \mathscr{D} \bigcup \mathcal{S}$ then we will compute separately the weak and strong exponents. We will first prove that for any point $x$ in $[0,1]$ which is not a maximum or a minimum of $F$ the two weak exponents vanish.

Proposition 19. Let $x \notin \mathscr{D} \bigcup \mathcal{S}$ and $X=(x, f(x))$. Then $E_{\Omega}^{w}(X)=E_{\Omega^{c}}^{w}(X)=0$.

Proof. We will prove first that we always have $E_{\Omega^{c}}^{w}(X)=0$ but will separate the proofs in cases $r(x)>1$ and $r(x)=1$. Then we will prove that $E_{\Omega}^{w}(X)=0$ and prove it separately for $s(x)>1$ and $s(x)=1$.

(i) Case $r(x)>1$. We follow the notations of Case 3 of Lemma 15; that is, one can find two subsequences $J_{n}$ and $J_{n}^{\prime}$ such that $J_{n}^{\prime} / J_{n}>1$ and $i_{J_{n}}(x)=i_{J_{n}^{\prime}+1}(x), i_{j}(x)+i_{J_{n}}(x)=1$ for $J_{n}<j<J_{n}^{\prime}+1$. Suppose without lose of generality that $i_{J_{n}+1}(x)=0$. Let $\tilde{x}_{n}=K_{J_{n}} / 2^{J_{n}}=\sum_{j=1}^{J_{n}} i_{j}(x) / 2^{j}$. Thus we have

$$
2^{-J_{n}^{\prime}-1} \leq x-\tilde{x}_{n} \leq 2^{-J_{n}^{\prime}}
$$

Since Case 3 of Lemma 15 holds, we get

$$
\begin{gathered}
A 2^{(1-\alpha) J_{n}^{\prime}} 2^{-J_{n}^{\prime}-1} \leq F_{J_{n}^{\prime}-1}(x)-F_{J_{n}^{\prime}-1}\left(\tilde{x}_{n}\right) \leq B 2^{(1-\alpha) J_{n}^{\prime}} 2^{-J_{n}^{\prime}}, \\
A^{\prime} 2^{-\alpha J_{n}^{\prime}} \leq F_{J_{n}^{\prime}-1}(x)-F_{J_{n}^{\prime}-1}\left(\tilde{x}_{n}\right) \leq B^{\prime} 2^{-\alpha J_{n}^{\prime}} .
\end{gathered}
$$

We have $F(x)=F_{J_{n}^{\prime}-1}(x)+\sum_{k=J_{n}^{\prime}}^{+\infty} 2^{-k \alpha} \Lambda\left(\tau^{k} x\right)$ and $F_{J_{n}^{\prime}-1}\left(\tilde{x}_{n}\right)=$ $F\left(\tilde{x}_{n}\right)$. Clearly $\sum_{k=J_{n}^{\prime}}^{+\infty} 2^{-k \alpha} \Lambda\left(\tau^{k} x\right) \geq 0$. Thus following (92) we have

$$
\begin{gathered}
A 2^{(1-\alpha) J_{n}^{\prime}} 2^{-J_{n}^{\prime}-1} \leq F(x)-F\left(\tilde{x}_{n}\right) \\
\leq B 2^{(1-\alpha) J_{n}^{\prime}} 2^{-J_{n}^{\prime}}+2^{-J_{n}^{\prime} \alpha} \sum_{k=0}^{+\infty} 2^{-k \alpha} \Lambda\left(\tau^{k+J_{n}^{\prime}} x\right) \\
A^{\prime} 2^{-\alpha J_{n}^{\prime}} \leq F(x)-F\left(\tilde{x}_{n}\right) \leq B^{\prime} 2^{-J_{n}^{\prime}}+2^{-\alpha J_{n}^{\prime}} F\left(\tau^{J_{n}^{\prime}} x\right) \\
A^{\prime} 2^{-\alpha J_{n}^{\prime}} \leq F(x)-F\left(\tilde{x}_{n}\right) \leq B^{\prime} 2^{-J_{n}^{\prime}}+2^{-\alpha J_{n}^{\prime}} F\left(\tau^{J_{n}^{\prime}} \frac{1}{3}\right) \\
A^{\prime} 2^{-\alpha J_{n}^{\prime}} \leq F(x)-F\left(\tilde{x}_{n}\right) \leq C 2^{-\alpha J_{n}^{\prime}}
\end{gathered}
$$

because the global maxima of $F$ are reached at points $1 / 3$ and $2 / 3$.

We can now apply the mean value theorem and get that for each $n \geq J_{0}$ we can find $\left.y_{n} \in\right] x-2^{-J_{n}^{\prime}}, x+2^{-J_{n}^{\prime}}$ [ such that $F(x)-F\left(y_{n}\right)=A^{\prime} \cdot 2^{-\alpha n} / 2$.

Thus using Lemma 10 we can conclude that $E_{\Omega^{c}}^{w}(X)=0$.

(ii) Case $r(x)=1$. Let $J_{n}$ be defined just as in Lemma 16, that is, that one can find $\delta>0, \delta^{\prime}>0$, and $J_{n}$ such that (54) is satisfied.

Following the definition of $r(x)$, for all $\gamma>0$, there exists $n_{0}$ such that for all $j \geq J_{n_{0}}\left|K_{j} 2^{-j}-x\right|>2^{-j(1+\gamma)}$. Thus in particular for all $n \geq n_{0}$ we have

$$
2^{-J_{n}}>\left|K_{J_{n}} 2^{-J_{n}}-x\right|>2^{-J_{n}(1+\gamma)} \text {. }
$$

Suppose on one hand $C_{J_{n}-1}(x) \geq 0$. Then choose $\tilde{x}_{n}=K_{J_{n}} 2^{-J_{n}}$ if $x \in] K_{J_{n}} 2^{-J_{n}}, 2^{-J_{n}}+K_{J_{n}} 2^{-J_{n}}\left[\right.$ (resp. $\tilde{x}_{n}=K_{J_{n}} 2^{-J_{n}}-2^{-J_{n}}$ if $x \in] K_{J_{n}} 2^{-J_{n}}-2^{-J_{n}}, K_{J_{n}} 2^{-J_{n}}[$ ).

We have obviously

$$
F_{J_{n}-1}(x)-F_{J_{n}-1}\left(\tilde{x}_{n}\right)=C_{J_{n}-1}(x)\left(x-\tilde{x}_{n}\right) \geq 0 .
$$

If we suppose on the other hand $C_{J_{n}-1}(x) \leq 0$, then we can choose in the same way a dyadic number $\tilde{x}_{n}=k / 2^{J_{n}}$ such that

$$
F_{J_{n}-1}(x)-F_{J_{n}-1}\left(\tilde{x}_{n}\right)=C_{J_{n}-1}(x)\left(x-\tilde{x}_{n}\right) \geq 0 .
$$

Together with (54) this yields in any of these cases that

$$
\begin{gathered}
\delta^{\prime} 2^{-\alpha J_{n}} \geq\left|F_{J_{n}-1}(x)-F_{J_{n}-1}\left(\tilde{x}_{n}\right)\right| \geq \delta 2^{-J_{n}(1+\gamma)} 2^{(1-\alpha) J_{n}}, \\
\delta^{\prime} 2^{-\alpha J_{n}} \geq F_{J_{n}-1}(x)-F_{J_{n}-1}\left(\tilde{x}_{n}\right) \geq \delta 2^{-J_{n}(1+\gamma)} 2^{(1-\alpha) J_{n}} .
\end{gathered}
$$

Since $\sum_{k=J_{n}^{\prime}}^{+\infty} 2^{-k \alpha} \Lambda\left(\tau^{k} x\right) \geq 0$ and $F_{J_{n}-1}\left(\tilde{x}_{n}\right)=F\left(\tilde{x}_{n}\right)$ we get

$$
\begin{aligned}
& \delta^{\prime} 2^{-\alpha J_{n}}+2^{-\alpha J_{n}} F\left(\frac{1}{3}\right) \geq F(x)-F\left(\tilde{x}_{n}\right) \\
& \geq F_{J_{n}-1}(x)-F_{J_{n}-1}\left(\widetilde{x}_{n}\right) \\
& \geq \delta 2^{-J_{n}(1+\gamma)} 2^{(1-\alpha) J_{n}}, \\
& C 2^{-\alpha J_{n}} \geq F(x)-F\left(\tilde{x}_{n}\right) \geq \delta 2^{-J_{n}(\gamma+\alpha)} .
\end{aligned}
$$


To get $E_{\Omega^{c}}^{w}(X)$ we only have to adapt the proof of Lemma 10 to the case $r_{n}=2^{-J_{n}}$. Suppose without loss of generality that $x<\tilde{x}_{n}$ (the other case can be treated in a similar way) and let $r_{n}=2^{-J_{n}}$ for $n \geq n_{0}$.

Indeed, since for $\gamma$ being small enough and for $n$ being large enough $2^{-J_{n}}$ is negligible in front of $2^{-(\alpha+\gamma) J_{n}}$ (what we denote $2^{-(\alpha+\gamma) J_{n}} \gg 2^{-J_{n}}$ ), following the mean value theorem we can find $\left.b_{n} \in\right] \min \left(x, \tilde{x}_{n}\right), \max \left(x, \tilde{x}_{n}\right)\left[\right.$ such that $b_{n}=$ $\sup \left\{u_{n} \in\right] x, \tilde{x}_{n}\left[, f\left(u_{n}\right)=f(x)-r_{n}\right\}$. For all $\left.t \in\right] b_{n}, \tilde{x}_{n}$ [, we have $f(t)<f(x)-r_{n}$. Thus following the same method as in Lemma 10 we can find $C>0$ such that

$$
C^{\prime} r_{n}^{2} \geq \operatorname{meas}\left(B\left(X, r_{n}\right) \bigcap \Omega^{c}\right) \geq C r_{n}^{(1+(\gamma / \alpha))+1} .
$$

This yields

$$
\begin{aligned}
2 & \leq \liminf _{n \rightarrow+\infty} \frac{\log \left(\text { meas }\left(B\left(X, r_{n}\right) \cap \Omega^{c}\right)\right)}{\log \left(r_{n}\right)} \\
& \leq \limsup _{n \rightarrow+\infty} \frac{\log \left(\operatorname{meas}\left(B\left(X, r_{n}\right) \cap \Omega^{c}\right)\right)}{\log \left(r_{n}\right)} \\
& \leq 2+\frac{\gamma}{\alpha} .
\end{aligned}
$$

Since $\gamma>0$ is arbitrary and $r_{n}$ is independent of $\gamma$, we have the result and $E_{\Omega^{c}}^{w}(X)=0$.

(iii) Case $s(x)>1$. Following Case 4, then one can find two subsequences $J_{n}$ and $J_{n}^{\prime}$ with $J_{n}^{\prime} / J_{n}>1$ for all $n$, such that $i_{j}(x)+i_{j+1}(x)=1$ for $J_{n}<j<J_{n}^{\prime}$ and $i_{J_{n}^{\prime}}(x)=i_{J_{n}^{\prime}+1}(x)$.

Suppose without losing generality that $i_{J_{n}^{\prime}}(x)=0$.

Let $\widetilde{X}_{n}$ such that $\widetilde{X}_{n}=\left(k_{J_{n}} / 2^{J_{n}}\right)+\left(2 / 3\left(2^{J_{n}}\right)\right)=$ $\sum_{j=1}^{J_{n}}\left(i_{j}(x) / 2^{j}\right)+\left(2 / 3\left(2^{J_{n}}\right)\right)$. We have clearly

$$
2^{-J_{n}^{\prime}-1} \leq-x+\widetilde{X}_{n} \leq 2^{-J_{n}^{\prime}} .
$$

Following the same sketch as in the proof with $r(x)>1$ we can say that using Case 4 of Lemma 15

$$
A^{\prime} 2^{-\alpha J_{n}^{\prime}} \leq F_{J_{n}^{\prime}-1}\left(\widetilde{X}_{n}\right)-F_{J_{n}^{\prime}-1}(x) \leq B^{\prime} 2^{-\alpha J_{n}^{\prime}}
$$

and since $2^{-\alpha J_{n}^{\prime}} F\left(\tau^{J_{n}^{\prime}} 1 / 3\right) \geq \sum_{k=J_{n}^{\prime}}^{+\infty} 2^{-k \alpha} \Lambda\left(\tau^{k} \widetilde{X}_{n}\right) \quad-$ $\sum_{k=J_{n}^{\prime}}^{+\infty} 2^{-k \alpha} \Lambda\left(\tau^{k} x\right) \geq 0$ we have indeed

$$
A^{\prime} 2^{-\alpha J_{n}^{\prime}} \leq F\left(\widetilde{X}_{n}\right)-F(x) \leq C 2^{-\alpha J_{n}^{\prime}} .
$$

Thus using the mean value theorem and Lemma 11 as in the previous case we conclude that $E_{\Omega}^{w}(X)=0$.

(iv) Case $s(x)=1$. Let $J_{n}$ be defined just as in Lemma 16, that is, that one can find $\delta>0, \delta^{\prime}>0$, and $J_{n}$ such that (54) is satisfied as well as Point 2 of Lemma 16.

Following the definition of $s(x)$, for all $\gamma>0$ there exists $n_{0}$ such that for all $j \geq J_{n_{0}}\left|m_{j}-x\right|>2^{-j(1+\gamma)}$. Thus in particular for all $n \geq n_{0}$ we have

$$
2^{-J_{n}}>\left|m_{J_{n}}-x\right|>2^{-J_{n}(1+\gamma)} .
$$

Suppose on one hand that $C_{J_{n}-1}(x)>0$. Then take $\widetilde{X}_{n}=$ $\left(K_{J_{n}} / 2^{J_{n}}\right)+\left(2 / 3\left(2^{J_{n}}\right)\right)$. Since $i_{J_{n}+1}(x)=0$ we have $F_{J_{n}-1}\left(\widetilde{X}_{n}\right)-$ $F_{J_{n}-1}(x)>0$.

Together with (54) this yields that

$$
\begin{aligned}
& \delta^{\prime} 2^{-\alpha J_{n}} \geq\left|F_{J_{n}-1}(x)-F_{J_{n}-1}\left(\widetilde{X}_{n}\right)\right| \geq \delta 2^{-J_{n}(1+\gamma)} 2^{(1-\alpha) J_{n}} \\
& \delta^{\prime} 2^{-\alpha J_{n}} \geq-F_{J_{n}-1}(x)+F_{J_{n}-1}\left(\widetilde{X}_{n}\right) \geq \delta 2^{-J_{n}(1+\gamma)} 2^{(1-\alpha) J_{n}} .
\end{aligned}
$$

The same computation used previously yields

$$
\begin{aligned}
\delta^{\prime} 2^{-\alpha J_{n}}+2^{-\alpha J_{n}} F\left(\frac{1}{3}\right) & \geq-F(x)+F\left(\widetilde{X}_{n}\right) \\
& \geq-F_{J_{n}-1}(x)+F_{J_{n}-1}\left(\widetilde{X}_{n}\right) \\
& \geq \delta 2^{-J_{n}(1+\gamma)} 2^{(1-\alpha) J_{n}}, \\
C 2^{-\alpha J_{n}} \geq-F(x) & +F\left(\widetilde{X}_{n}\right) \geq \delta 2^{-J_{n}(\gamma+\alpha)} .
\end{aligned}
$$

To get $E_{\Omega}^{w}(X)$ we only have to adapt the proof of Lemma 11 in the same way we adapt the one of Lemma 10 in the case $r(x)=1$.

Thus taking $r_{n}=2^{-J_{n}}$ and following the same method used previously we can find $C>0$ such that

$$
C^{\prime} r_{n}^{2} \geq \operatorname{meas}\left(B\left(X, r_{n}\right) \bigcap \Omega\right) \geq C 2^{-J_{n}(1+(\gamma / \alpha))} 2^{-J_{n}} .
$$

This yields

$$
\begin{aligned}
2 & \leq \liminf _{n \rightarrow+\infty} \frac{\log \left(\operatorname{meas}\left(B\left(X, r_{n}\right) \cap \Omega\right)\right)}{\log \left(r_{n}\right)} \\
& \leq \limsup _{n \rightarrow+\infty} \frac{\log \left(\operatorname{meas}\left(B\left(X, r_{n}\right) \cap \Omega\right)\right)}{\log \left(r_{n}\right)} \\
& \leq 2+\frac{\gamma}{\alpha} .
\end{aligned}
$$

Since $\gamma>0$ is arbitrary, we have the result and $E_{\Omega}^{w}(X)=0$.

Hence Proposition 19 holds.

For what concerns the strong accessibility exponent we have the following result.

Proposition 20. Suppose $x \notin \mathscr{D} \bigcup \mathcal{S}$ and let $X_{0}=(x, F(x))$. Then we have the following.

(1) If $r(x)>1 / \alpha$ then $E_{\Omega^{c}}^{s}\left(X_{0}\right)=(1 / \alpha)-1$.

(2) If $s(x)>1 / \alpha$ then $E_{\Omega}^{s}\left(X_{0}\right)=(1 / \alpha)-1$.

(3) Let $D_{\alpha}$ the set of $x \notin \mathscr{D} \bigcup \mathcal{S}$ such that $r(x)>1 / \alpha$ and $s(x)>1 / \alpha$. Then the Hausdorff dimension of $D_{\alpha}$ is $\alpha$.

Proof. (1) Let us prove Point 1. Since $r(x)>1 / \alpha$ and following Point 3 of Lemma 15, for $\delta>0$ such that $r(x)-\delta>1 / \alpha$, we can find $J_{n}^{\prime}$ and $J_{n}$ such that

(i) $x_{n}=K_{J_{n}} 2^{-J_{n}}$ and $\left|x-x_{n}\right| \leq 2^{-J_{n}^{\prime}}$;

(ii) $\left|F(x)-F\left(x_{n}\right)\right| \leq 2^{-\alpha J_{n}^{\prime}}$. 
Since we can choose $J_{n}^{\prime}$ such that $2^{-J_{n}^{\prime}} \leq C 2^{(r(x)-\delta) J_{n}}$ (see the proof of Point 3 of Lemma 15), then $\left|x-x_{n}\right|$ is negligible in front of $2^{-J_{n}}$ (what we denote $\left|x-x_{n}\right| \ll 2^{-J_{n}}$ ) and $\mid F(x)-$ $F\left(x_{n}\right) \mid \leq 2^{-\alpha J_{n}^{\prime}} \ll 2^{-J_{n}}$.

Thus we can choose a constant $C$ such that with $\rho_{n}=$ $C 2^{-J_{n}}$ and $B\left(X_{0}, \rho_{n} / 2\right) \subset B\left(\left(x_{n}, F\left(x_{n}\right)\right), \rho_{n}\right)$. Following the proof of Proposition 17 and more precisely (73) we have

$$
\begin{aligned}
\operatorname{meas} & \left(B\left(X, \frac{\rho_{n}}{2}\right) \bigcap \Omega^{c}\right) \\
& \leq \operatorname{meas}\left(B\left(\left(K_{J_{n}} 2^{-J_{n}}, F\left(K_{J_{n}} 2^{-J_{n}}\right), \rho_{n}\right) \bigcap \Omega^{c}\right)\right) \\
& \leq C \rho_{n}^{1+1 / \alpha} .
\end{aligned}
$$

This yields $E_{\Omega^{c}}^{s}(X) \geq(1 / \alpha)-1$.

Since $E_{\Omega^{c}}^{s}(X) \leq(1 / \alpha)-1$, we get $E_{\Omega^{c}}^{s}(X)=(1 / \alpha)-1$.

(2) We follow exactly the same proof as previously replacing $x_{n}$ by $\widetilde{X}_{n}$ the sequence of local maxima defined in the proof of Point 4 of Lemma 15.

(3) We follow here the results proved by [18] and summarized in [19] for our special case. Indeed recall the definition given in [18] of an ubiquitous system in a real interval of R.

Definition 21. Let $U$ be a real open interval. Let $\left(x_{i}\right)_{i \geq 1}$ be points in $U$ and let $\left(r_{i}\right)_{i \geq 1}$ be a sequence of positive real numbers such that $\lim _{i \rightarrow \infty} r_{i}=0$. The family $\left(x_{i}, r_{i}\right)_{i \geq 1}$ is a homogeneous ubiquitous system in $U$ if the set $\lim _{\sup _{i}} B\left(x_{i}, r_{i}\right)$ is of full Lebesgue measure in $U$. result.

Theorem D of [19] proved in [18] yields the following

Theorem 22. Let $\tau$ be a real number with $\tau \geq 1$. With the above notations if the families $\left(x_{i}, r_{i}\right)_{i \geq 1}$ and $\left(x_{i}^{\prime}, r_{i}^{\prime}\right)_{i \geq 1}$ are two homogeneous ubiquitous systems in $U$, then the Hausdorff dimension of the set $\lim \sup B\left(x_{i}, r_{i}^{\tau}\right) \bigcap \lim \sup B\left(\tilde{x}_{j}, \widetilde{r}_{j}^{\tau}\right)$ is at least equal to $1 / \tau$.

Let $U=] 0,1\left[\right.$ and consider $\mathscr{K}_{1}=\left\{\left(k / 2^{j}, 2^{-j}\right), k \in \mathbb{N}, 0<\right.$ $\left.k<2^{j}, j \geq 1\right\}$. It is a countable set and can be written as $\mathscr{K}_{1}=\left\{\left(x_{i}, r_{i}\right), i \geq 1\right\}$ with $x_{i}$ a dyadic number for all $i \geq 1$. Let $\mathscr{K}_{2}=\left\{(x, r), x \in \mathcal{S}, r=2^{-j} / 3\right.$ for $\left.j \geq 1\right\}$. It is again a countable set and we can rewrite it as $\mathscr{K}_{2}=\left\{\left(\tilde{x}_{i}, \widetilde{r}_{i}\right), i \geq 1\right\}$ with $\tilde{x}_{i} \in \mathcal{S}$ for all $i \geq 1$.

It is clear that $\lim \sup B\left(x_{i}, r_{i}\right)$ and $\lim \sup B\left(\tilde{x}_{i}, \tilde{r}_{i}\right)$ are of full Lebesgue measure.

Remark then that $D_{\alpha}=\lim \sup B\left(x_{i}, r_{i}^{\tau}\right) \bigcap \lim \sup B\left(\tilde{x}_{i}\right.$, $\left.\tilde{r}_{i}^{\tau}\right)$ with $\tau=1 / \alpha$. Since $D_{\alpha} \subset \bigcup_{j \geq J, 0 \leq k \leq 2^{j}} B\left(x_{i}, r_{i}^{\tau}\right)$ the Hausdorff dimension of $D_{\alpha}$ is less than or equal to $\alpha$. We apply Theorem 22 and we find it is exactly $\alpha$.

4.4. Proof of Theorem 8. Propositions 17, 18, 19, and 20 achieve the proof of Theorem 8 .

\section{Conflict of Interests}

The authors declare that there is no conflict of interests regarding the publication of this paper.

\section{Acknowledgments}

The first author would like to extend his sincere appreciation to the Deanship of Scientific Research at King Saud University for its funding Research Group no. RG-1435-063. The authors are very thankful to A. Gaudillière for very fruitful discussions, Y. Heurteaux for stimulating discussions that helped them to obtain Lemma 10, and Y. Bugeaud for sharing with them key references.

\section{References}

[1] P. C. Allaart and K. Kawamura, "The Takagi function: a survey," Real Analysis Exchange, vol. 37, no. 1, pp. 1-54, 2011.

[2] K. Knopp, "Ein einfaches Verfahren zur Bildüng stetiger nirgends differenzierbarer Funktionen," Mathematische Zeitschrift, vol. 2, no. 1-2, pp. 1-26, 1918.

[3] Z. Ciesielski, "On the isomorphisms of the spaces $H$, and $M$," Bull. Acad. Polon. Sci. Skr. Sci. Math. Astronom. Phys., vol. 8, pp. 217-222, 1960.

[4] A.-P. Calderón and A. Zygmund, "Local properties of solutions of elliptic partial differential equations," Studia Mathematica, vol. 20, pp. 171-225, 1961.

[5] S. Jaffard and B. B. Mandelbrot, "Local regularity of nonsmooth wavelet expansions and application to the Polya function," Advances in Mathematics, vol. 120, no. 2, pp. 265-282, 1996.

[6] C. Tricot, Curves and Fractal Dimension, Springer, 1995.

[7] Z. Ciesielski, "Fractal functions and Schauder bases," Computers \& Mathematics with Applications, vol. 30, no. 3-6, pp. 283-291, 1995.

[8] Z. Ciesielski, "Spline orthogonal systems and fractal functions," Acta Mathematica Hungarica, vol. 68, no. 4, pp. 287-293, 1995.

[9] S. Jaffard, "Oscillation spaces: properties and applications to fractal and multifractral functions," Journal of Mathematical Physics, vol. 39, no. 8, pp. 4129-4141, 1998.

[10] A. Kamont and B. Wolnik, "Wavelet expansions and fractal dimensions," Constructive Approximation, vol. 15, no. 1, pp. 97108, 1999.

[11] F. Ledrappier, "On the dimension of some graphs," Contemporary Mathematics, vol. 135, pp. 285-293, 1992.

[12] B. Solomyak, "On the random series $\sum \pm \lambda^{n}$ (an Erdös problem)," Annals of Mathematics, vol. 142, no. 3, pp. 611-625, 1995.

[13] S. Jaffard and C. Mélot, "Wavelet analysis of fractal boundaries. Part 1: local exponents," Communications in Mathematical Physics, vol. 258, no. 3, pp. 513-539, 2005.

[14] Y. Heurteaux and S. Jaffard, "Multifractal analysis of images: new connexions between analysis and geometry," in Imaging for Detection and Identification, NATO Security through Science Series, pp. 169-194, Springer, 2007.

[15] C. Tricot, "General Hausdorff functions, and the notion of onesided measure and dimension," Arkiv för Matematik, vol. 48, no. 1, pp. 149-176, 2010.

[16] B. Dubuc and S. Dubuc, "Error bounds on the estimation of fractal dimension," SIAM Journal on Numerical Analysis, vol. 33, no. 2, pp. 602-626, 1996. 
[17] S. Dubuc and A. Elqortobi, "Le maximum de la fonction de Knopp," INFOR, vol. 26, no. 4, pp. 311-323, 1990.

[18] A. Durand, "Sets with large intersection and ubiquity," Mathematical Proceedings of the Cambridge Philosophical Society, vol. 144, no. 1, pp. 119-144, 2008.

[19] M. Amou and Y. Bugeaud, "Exponents of Diophantine approximation and expansions in integer bases," Journal of the London Mathematical Society. Second Series, vol. 81, no. 2, pp. 297-316, 2010. 


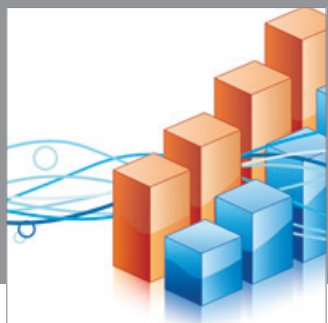

Advances in

Operations Research

mansans

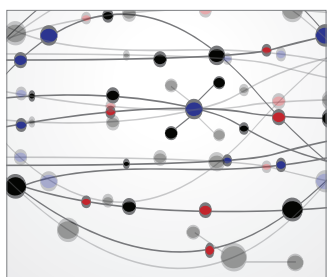

The Scientific World Journal
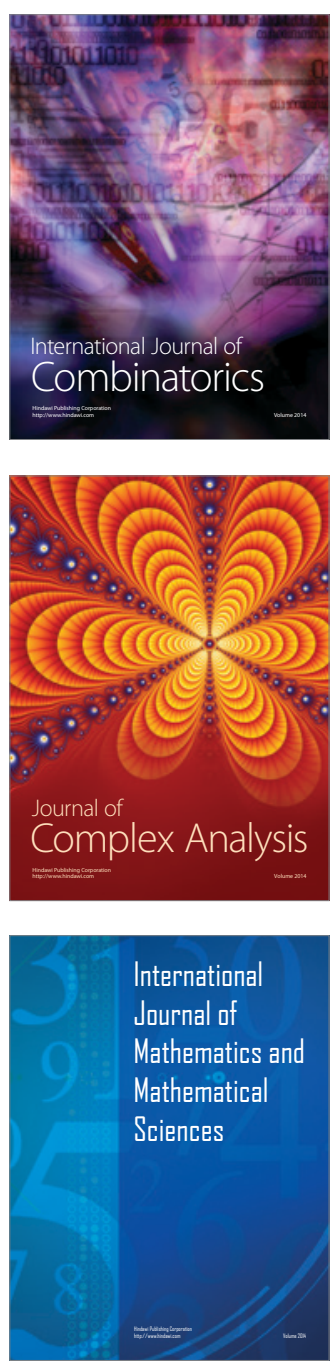
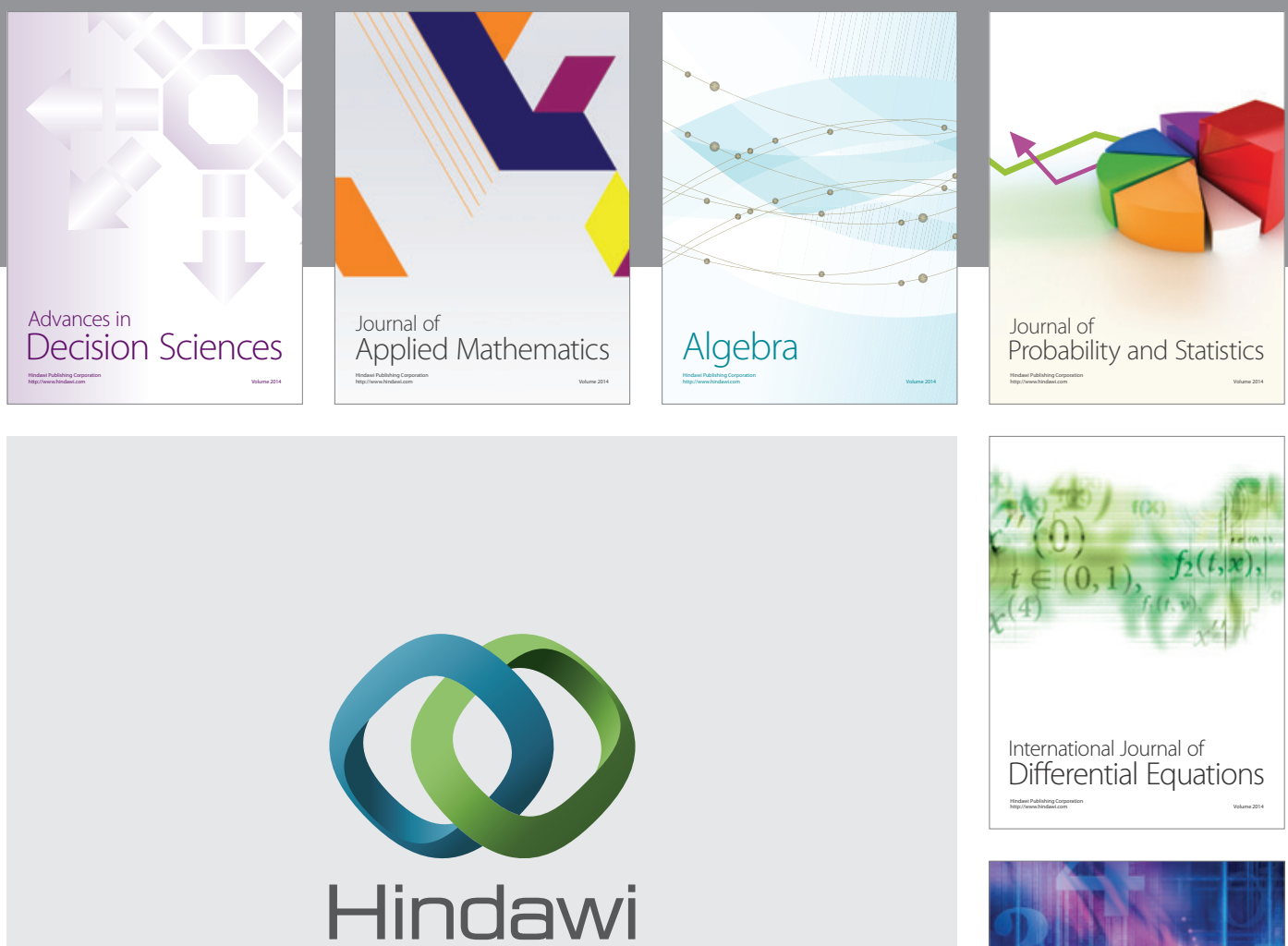

Submit your manuscripts at http://www.hindawi.com
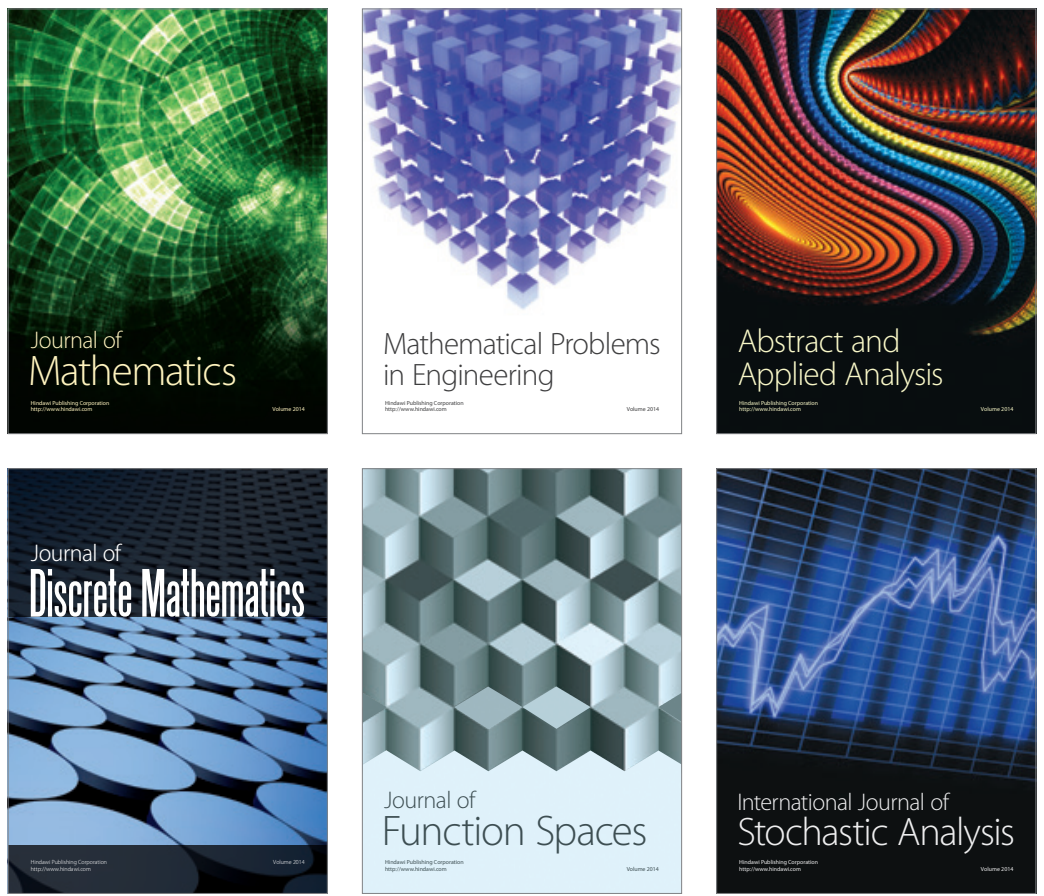

Journal of

Function Spaces

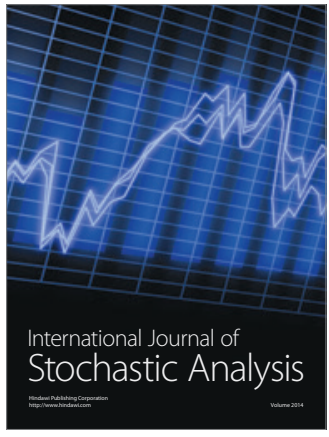

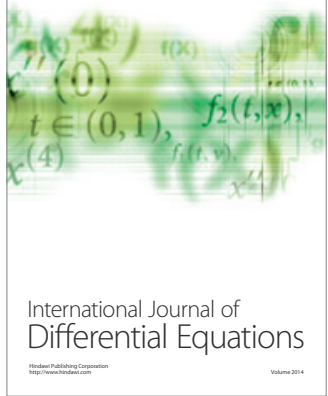
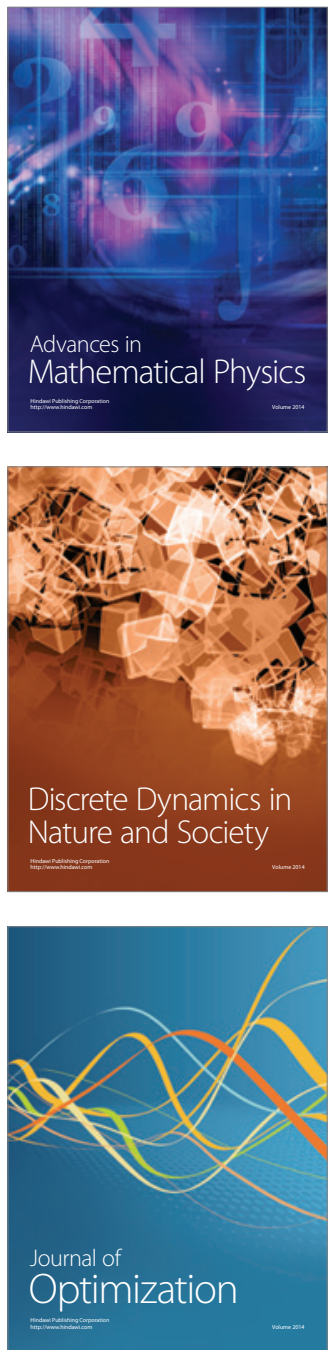